\title{
DIRAC MANIFOLDS
}

\author{
THEODORE JAMES COURANT
}

\begin{abstract}
A Dirac structure on a vector space $V$ is a subspace of $V$ with a skew form on it. It is shown that these structures correspond to subspaces of $V \oplus V^{*}$ satisfying a maximality condition, and having the property that a certain symmetric form on $V \oplus V^{*}$ vanishes when restricted to them. Dirac structures on a vector space are analyzed in terms of bases, and a generalized Cayley transformation is defined which takes a Dirac structure to an element of $O(V)$. Finally a method is given for passing a Dirac structure on a vector space to a Dirac structure on any subspace.

Dirac structures on vector spaces are generalized to smooth Dirac structures on a manifold $P$, which are defined to be smooth subbundles of the bundle $T P \oplus T^{*} P$ satisfying pointwise the properties of the linear case. If a bundle $L \subset T P \oplus T^{*} P$ defines a Dirac structure on $P$, then we call $L$ a Dirac bundle over $P$. A 3-tensor is defined on Dirac bundles whose vanishing is the integrability condition of the Dirac structure. The basic examples of integrable Dirac structures are Poisson and presymplectic manifolds; in these cases the Dirac bundle is the graph of a bundle map, and the integrability tensors are $[B, B]$ and $d \Omega$ respectively. A function $f$ on a Dirac manifold is called admissible if there is a vector field $X$ such that the pair $(X, d f)$ is a section of the Dirac bundle $L$; the pair $(X, d f)$ is called an admissible section. The set of admissible functions is shown to be a Poisson algebra.

A process is given for passing Dirac structures to a submanifold $Q$ of a Dirac manifold $P$. The induced bracket on admissible functions on $Q$ is in fact the Dirac bracket as defined by Dirac for constrained submanifolds.
\end{abstract}

\section{INTRODUCTION}

The underlying structure in any formulation of Hamiltonian systems is a general Poisson algebra, an associative commutative algebra with a Lie bracket operation $\{$,$\} satisfying the Leibniz identity (i.e., that \{f$,$\} is a derivation:$ $\{f, g h\}=g\{f, h\}+\{f, g\} h)$; see, for example, Sniatycki and Weinstein [1983] or Vinogradov and Krasilshchik [1975].

In symplectic and Poisson geometry on a smooth manifold $P$, the Poisson algebra is $C^{\infty}(P)$ and the bracket $\{$,$\} is given by a smooth bivector field \Lambda$ on $P$ satisfying $[\Lambda, \Lambda]=0$, i.e., the Schouten bracket of $\Lambda$ with itself is zero (for a discussion of Poisson manifolds see Weinstein [1983] and the references

Received by the editors August 22, 1988.

1980 Mathematics Subject Classification (1985 Revision). Primary 70H99, 53A17.

Key words and phrases. Poisson manifold, symplectic manifold, Dirac brackets, constrained dynamics, Lie algebroid. 
therein). In the symplectic case the bivector field is (minus) the inverse of the symplectic form.

Presymplectic geometry is the study of the Poisson algebra of functions on $P$ associated to a closed 2-form $\Omega$ in the following way: we say that a vector field $X$ is generated by the form $\Omega$ and the function $H$ if

$$
X\lrcorner \Omega=d H ;
$$

if the pair $(X, d H)$ satisfies equation (1), we write $X=X_{H}$ and call $H$ a Hamiltonian function for $X_{H}$. We call the kernel of the bundle map $\Omega: T P \rightarrow$ $T^{*} P$ the characteristic distribution of the 2 -form $\Omega$. Thus $X_{H}$ is defined only up to vector fields in the characteristic distribution of $\Omega$; these are called gauge vector fields. If $Z$ is a gauge vector field we assume that the solutions of equation (1) given by $X_{H}$ and $X_{H}+Z$ describe the same dynamics, i.e., the ambiguity in the definition of $X_{H}$ has no physical significance. For discussion of gauge freedom in Hamiltonian systems, see Gotay and Nester [1979], or Gotay [1983].

The Poisson algebra associated to $\Omega$ is given by the set of functions $H$ for which equation (1) has a solution $X_{H}$, and the bracket on this set is defined as

$$
\{H, G\}=\Omega\left(X_{H}, X_{G}\right)=X_{G} \cdot H .
$$

Notice that this bracket is well defined, even though $X_{H}$ is not. The Jacobi and Leibniz identities for this bracket follow from the closedness of $\Omega$. Therefore equation (2) defines a Poisson algebra. We say that this algebra is generated by the presymplectic structure $\Omega$; see for example Pnevmatikos [1979, 1984, 1985], Lichnerowicz [1977], or Martinet [1970].

Assume now that $(P, \Omega)$ is symplectic. If $i_{Q}: Q \rightarrow P$ is the inclusion map of an arbitrary submanifold, we obtain a presymplectic structure $\Omega_{Q}$ on $Q$, namely

$$
\Omega_{Q}=i_{Q}^{*} \Omega .
$$

Consider now a submanifold $Q$ in a Poisson manifold $P . P$ has a singular foliation whose leaves are symplectic manifolds; therefore $Q$ is stratified by sets which are the intersection of $Q$ with the leaves of $P$ (for simplicity we assume that this is a foliation of $Q$ ). By the remarks preceding formula (3), each leaf of this foliation is presymplectic with closed 2-form equal to the restriction to $Q$ of the symplectic 2 -form on the leaf in $P$. Thus we have a manifold $Q$ foliated by presymplectic leaves. This situation arises, e.g., in the case of an equivariant momentum map $J: P \rightarrow \mathfrak{g}^{*}$; we consider the level set $Q=J^{-1}(0)$ : in this case $Q$ has presymplectic leaves whose characteristic distribution is given by the velocity vectors of the action of $G$ on $J^{-1}(0)$. The quotient manifold $J^{-1}(0) / G$ is a Poisson manifold. However a new structure is needed to describe $J^{-1}(0)$; it will turn out that $J^{-1}(0)$ is an integrable Dirac manifold, the main structure defined in this paper. This example is analyzed in $\S 3$ (see also Marsden and Ratiu [1985]). 
For simplicity we now assume that $(P, \Omega)$ is symplectic. As observed by Dirac, it is necessary to modify the Poisson bracket $\{,\}_{P}$ when one is constrained to a symplectic submanifold $Q$, i.e., a submanifold such that $\Omega_{Q}$ is a symplectic form. Suppose that $Q$ is given locally by constraints:

$$
Q=\left\{x \in P \mid \varphi^{\alpha}(x)=0\right\} \text { for independent functions } \varphi^{\alpha} .
$$

Let $c^{\alpha \beta}$ denote the matrix of brackets $\left\{\varphi^{\alpha}, \varphi^{\beta}\right\}$; Dirac showed that the matrix $c^{\alpha \beta}$ is necessarily invertible, and that the induced bracket $\{,\}_{Q}$ is given by

$$
\{f, g\}_{Q}=\{f, g\}_{P}-\left\{f, \varphi^{\alpha}\right\}_{P} c_{\alpha \beta}\left\{\varphi^{\beta}, g\right\}_{P},
$$

where $c_{\alpha \beta}$ denotes the inverse matrix of $c^{\alpha \beta}$. Equation (5) is called the Dirac bracket formula and it is to be interpreted as follows: $f$ and $g$ are functions on $P$, and the left-hand side is their bracket in $Q$ when they are restricted to $Q$; the right-hand brackets are in $P$, and equality occurs on $Q$. Thus we get a Poisson algebra on $C^{\infty}(Q)$. We may also choose any functions $f$ and $g$ on $Q$, extend them to functions on $P$, and apply equation (5); Dirac showed that equation (5) is independent of the choice of extension. For a general discussion of Dirac brackets see Dirac [1964], Regge, Hansen, and Teitelboim [1976], or Sniatycki [1974]; see also Marsden and Ratiu [1985], and Oh [1986] for an application of this formula to transverse Poisson structures.

In general, the form $\Omega_{Q}$ is degenerate, and (5) does not apply to all functions on $Q$. However, we may still define a Poisson algebra, namely the algebra of functions whose differentials annihilate $\operatorname{ker} \Omega_{Q}$.

This paper presents a unified approach to the geometry of Hamiltonian vector fields and their underlying Poisson algebras. The approach is based on concepts introduced in Guillemin and Sternberg [1977] for symmetric bilinear forms, applied here to skew symmetric forms (either covariant or contravariant). We define tensorial objects which correspond to brackets on subalgebras of functions. These objects are subbundles $L \subset T P \oplus T^{*} P$, and in the cases of Poisson structures and presymplectic structures, are the graphs of bundle maps $T^{*} P \rightarrow T P$ and $T P \rightarrow T^{*} P$ respectively; in these two cases integrability is defined as the vanishing of a 3-tensor, namely $[\Lambda, \Lambda]$ (the Schouten bracket of $\Lambda$ with itself) or $d \Omega$ respectively.

In general, we get a skew bivector on the quotient $T P / L \cap T P$, which gives us a bracket on the algebra of functions whose differentials annihilate $L \cap T P$; the distribution $L \cap T P$ in $T P$ is called the characteristic distribution. We present a general integrability condition, namely the vanishing of a 3-tensor on $L$, which implies that this bracket is a Poisson bracket. Thus these functions, i.e., those "constant along $L \cap T P$ ", form a Poisson algebra.

Consider now the distribution $\rho(L) \subset T P$, where $\rho$ is the projection of $T P \oplus T^{*} P$ onto $T P$. We define a 2 -form $\Omega_{L}: \rho(L) \rightarrow \rho(L)^{*}$ whose characteristic distribution is $L \cap T P \subset \rho(L)$. The vanishing of the integrability 3-tensor implies the integrability of $\rho(L)$ as a singular distribution and the closedness 
of the 2-form $\Omega_{L}$ on each leaf. Thus an integrable Dirac manifold is "foliated" by presymplectic "leaves".

The distributions $\rho(L)$ and $L \cap T P$ are generally not smooth subbundles of $T P$ since their dimensions do not have to be everywhere constant: locally, $\rho(L)$ is maximal on an open dense set, and $L \cap T P$ is minimal on an open dense set (not necessarily the same set). At best they will be integrable in the sense of Sussman: there is a maximal integral submanifold through every point; this is called the maximal integral manifold property. To illustrate this idea, suppose that a distribution $\Delta$ is given as the span of a collection of vector fields $X_{1}, \ldots, X_{n}$; we do not assume that $\Delta$ has constant dimension since we allow the vector fields $X_{i}$ to become linearly dependent (in the case of constant dimension, involutivity establishes the maximal integral manifold property; this is the classical theorem of Frobenius). Sussman has proved that $\Delta$ satisfies the maximal integral manifold property if and only if there are smooth functions $c_{i j}^{k}$ such that:

$$
\left[X_{i}, X_{j}\right]=c_{i j}^{k} X_{k} .
$$

For a discussion of singular distributions and their integrability see Sussman [1973] and Dazord [1985].

A sufficient condition is given in $\S 3$ for a submanifold $Q$ of a Dirac manifold $P$ to inherit a Dirac structure, namely that $L \cap\left(T Q \oplus T^{*} P\right)$ is a subbundle of $T P \oplus T^{*} P$. In this case we may construct a bundle $L_{Q} \subset\left(T Q \oplus T^{*} Q\right)$, which is again a Dirac structure. In $\S 3$ this process is applied to the problem of transverse structures, in the Poisson and Dirac settings.

A useful example of an integrable Dirac structure is provided by the singular Poisson structure on $\mathbb{R}^{3}$ given in coordinates $(x, y, z)$ by

$$
\{x, y\}=\frac{1}{z}, \quad\{x, z\}=0, \quad\{y, z\}=0 .
$$

This bracket gives us Hamiltonian vector fields

$$
X_{x}=-\frac{1}{z} \frac{\partial}{\partial y}, \quad X_{y}=\frac{1}{z} \frac{\partial}{\partial x}
$$

where are singular at $z=0$. We may rewrite this singular Poisson structure as a Dirac structure which is smooth even at $z=0$; since a Dirac structure is a bundle it is determined by local bases of sections, in this case

$$
\left(\frac{\partial}{\partial y},-z d x\right),\left(\frac{\partial}{\partial x}, z d y\right),(0, d z) .
$$

Thus we have a Dirac structure on $\mathbb{R}^{3}$ whose leaves are the planes $z=$ constant, and whose 2 -forms (on the leaves) are given by $\Omega=z d x \wedge d y$. The singular Poisson bracket given by (7) represents the averaged bracket in the problem of guiding center motion in the plane; for a discussion of this problem see Littlejohn [1979, 1981] or Omohundro [1984, 1985]. 


\section{LiNEAR Dirac STRUCTURES}

1.1. Dirac structures on a vector space. Let $V$ be a vector space. There are two natural pairings on $V \oplus V^{*}$, one symmetric and one skew symmetric, defined by

$$
\begin{aligned}
& \left\langle(x, y),\left(x^{\prime}, y^{\prime}\right)\right\rangle_{+}=\frac{1}{2}\left(\left\langle y \mid x^{\prime}\right\rangle+\left\langle x \mid y^{\prime}\right\rangle\right), \\
& \left\langle(x, y),\left(x^{\prime}, y^{\prime}\right)\right\rangle_{-}=\frac{1}{2}\left(\left\langle y \mid x^{\prime}\right\rangle-\left\langle x \mid y^{\prime}\right\rangle\right),
\end{aligned}
$$

and $(x, y),\left(x^{\prime}, y^{\prime}\right) \in V \oplus V^{*}$.

Definition 1.1.1. A Dirac structure on a vector space $V$ is a subspace $L \subset$ $V \oplus V^{*}$ which is maximally isotropic under the plus pairing $\langle,\rangle_{+}$.

We will see later that the dimension of a Dirac structure on $V$ is the dimension of $V$.

Example 1.1.2. Let $A: V \rightarrow V^{*}$ be a skew symmetric linear map, i.e., $A^{*}=-A$ with the identification of $V$ with $V^{* *}$. Then $\operatorname{graph}(A) \subset V \oplus V^{*}$ is isotropic under $\langle,\rangle_{+}$since $A$ satisfies

$$
\left\langle A x \mid x^{\prime}\right\rangle+\left\langle A x^{\prime} \mid x\right\rangle=0 .
$$

A dimension count shows that graphs of maps are maximally isotropic, so graph $(A)$ is a Dirac structure on $V$.

Example 1.1.3. Let $B: V^{*} \rightarrow V$ be skew symmetric. Then by the same reasoning as Example 1.1.2, we see that $\operatorname{graph}(B)$ is a Dirac structure on $V$.

We may think of these example as the presymplectic and Poisson cases of Dirac structures on vector spaces.

Now let $\rho$ and $\rho^{*}$ be the projections from $V \oplus V^{*}$ onto $V$ and $V^{*}$ respectively, and let $L$ denote a Dirac structure on $V$. Then $\left.\operatorname{ker} \rho\right|_{L}=L \cap V^{*}$ and $\left.\operatorname{ker} \rho^{*}\right|_{L}=L \cap V$. We claim that

$$
\rho(L)=L \cap V^{*} \text { and } \rho^{*}(L)=(L \cap V)^{\circ},
$$

where $W^{\circ}$ means the annihilator of $W$ (note that $L \cap V$ may be thought of as a subspace of either $V \oplus V^{*}$ or $V$, as suits the circumstance; similarly for $\left.L \cap V^{*}\right)$. To prove the claim, observe that

$$
\left\langle\rho^{*}(L) \mid \rho(L \cap V)\right\rangle=-\left\langle\rho^{*}(L \cap V) \mid \rho(L)\right\rangle=0,
$$

so clearly $\rho^{*}(L) \subset(L \cap V)^{\circ}$, and now a dimension count gives us equations (1.1.3), which we will refer to as the characteristic equations of a Dirac structure.

Notice that in the two examples, we have transversality of $L$ with one of the two summands, $V$ or $V^{*}$, whereas equations (1.1.3) describe structures which may have nonzero intersection with each summand.

Now consider the subspace $E=\rho(L) \subset V$. Define $\Omega(\rho(x))=\left.\rho^{*}(x)\right|_{E}$; this gives a map $\Omega: E \rightarrow E^{*}$ which is skew symmetric since $\left\langle\rho^{*}(x) \mid \rho(y)\right\rangle+$ $\left\langle\rho^{*}(y) \mid \rho(x)\right\rangle=0$ for all $x, y \in L$. To see that $\Omega$ is well defined, suppose we 
have $x, x^{\prime} \in L$ such that $\rho(x)=\rho\left(x^{\prime}\right)$; we will show that $\left.\rho^{*}(x)\right|_{E}=\left.\rho^{*}\left(x^{\prime}\right)\right|_{E}$. In fact, since $\rho(x)=\rho\left(x^{\prime}\right), x-\left.x^{\prime} \in \operatorname{ker} \rho\right|_{L}$, so $x-x^{\prime} \in L \cap V^{*}$; therefore $\rho^{*}\left(x-x^{\prime}\right) \in \rho(L)^{\circ}=E^{\circ}$, which says exactly that $\left.\rho^{*}(x)\right|_{E}=\left.\rho^{*}\left(x^{\prime}\right)\right|_{E}$. Notice that $L \cap V \subset E$ is the kernel of $\Omega$.

In the same way we also get a subspace $\rho^{*}(L) \subset V^{*}$, and a skew symmetric map $\Pi: \rho^{*}(L) \rightarrow \rho^{*}(L)^{*}$ whose kernel is $L \cap V^{*}$. We have $\rho^{*}(L)^{*}=$ $V / \rho^{*}(L)^{\circ}=V / L \cap V$ or $\rho^{*}(L)=(V / L \cap V)^{*}$, so this gives us $\Pi:(V / L \cap V)^{*} \rightarrow$ $V / L \cap V$. Thus if we consider $\Omega$ to be a 2 -form on $E, \Pi$ is a bivector on the quotient $V / L \cap V=V / \operatorname{ker} \Omega$.

Let us summarize:

Proposition 1.1.4. A Dirac structure $L \subset V \oplus V^{*}$ induces a skew form on the subspace $\rho(L) \subset V$; the kernel of this form is $L \cap V \subset \rho(L)$. At the same time, a Dirac structure induces a skew bivector on the quotient $V / L \cap V$.

Furthermore, given a skew form $\Omega$ on a subspace of $E \subset V$, we may reconstruct an associated Dirac structure as follows: since we are given the skew form, we know its kernel; therefore we have the spaces $L \cap V$ and $\rho(L)=E$, and this determines the spaces $\rho^{*}(L)$ and $L \cap V^{*}$. We may define $L=\left\{(x, y) \mid x \in E, y \in V^{*}\right.$ and $\left.\left.y\right|_{E}=\Omega(x)\right\} ; L$ is clearly isotropic under the symmetric pairing on $V \oplus V^{*}$, and its dimension is the dimension of $V$, since it contains subspaces of the form $\left(0, L \cap V^{*}\right)$ and $(E, \Omega(E))$. The fact that maximal isotropy occurs in this dimension will be shown in this section. Therefore $L$ is a Dirac structure on $V$.

Proposition 1.1.5. A Dirac structure on a vector space is equivalently defined as a subspace together with a skew form on the subspace.

1.2. Computations in a basis. Let us choose a basis for a Dirac structure $L$. This is the same as giving maps a: $\mathbb{R}^{n} \rightarrow V$ and $\mathbf{b}: \mathbb{R}^{n} \rightarrow V^{*}$, so that the basis becomes $\left(\mathbf{a} e_{1}, \mathbf{b} e_{1}\right), \ldots,\left(\mathbf{a} e_{n}, \mathbf{b} e_{n}\right)$. Notice that for these to span an $n$ dimensional space, we must have:

$$
\text { ker } \mathbf{a} \cap \operatorname{ker} \mathbf{b}=\{0\} .
$$

Now the isotropy of $L$ tells us that $\mathbf{a}^{*} \mathbf{b}+\mathbf{b}^{*} \mathbf{a}=0$, i.e., the map $\mathbf{a}^{*} \mathbf{b}: \mathbb{R}^{n} \rightarrow$ $\mathbb{R}^{n *}$ is skew symmetric. Notice that if $\mathbf{a}$ is invertible, we may use it to identify $V$ with $\mathbb{R}^{n}$ so that b becomes a $\mathbf{b}^{\prime}: V \rightarrow V^{*}$; thus $L$ is the graph of $\mathbf{b}^{\prime}$. Similarly, if $\mathbf{b}$ is invertible, $L$ is the graph of $\mathbf{a}^{\prime}: V^{*} \rightarrow V$.

Suppose now that we have a pair of maps $\mathbf{a}, \mathbf{b}$ such that

$$
\mathbf{a}^{*} \mathbf{b}+\mathbf{b}^{*} \mathbf{a}=0
$$

and

$$
\operatorname{ker} \mathbf{a} \cap \operatorname{ker} \mathbf{b}=\{0\} .
$$

Consider the set $\left\{(\mathbf{a} x, \mathbf{b} x) \in V \oplus V^{*}\right\}$. It is clearly isotropic under the symmetric pairing on $V \oplus V^{*}$, and $\operatorname{ker} \mathbf{a} \cap \operatorname{ker} \mathbf{b}=\{0\}$ implies that it has the dimension of $V$. Therefore it is an isotropic subspace of maximal dimension, and consequently is a Dirac structure on $V$. 
Definition 1.2.1. A pair of maps (a, b) satisfying equations (1.2.1) and (1.2.2) is called a basis representation of a Dirac structure.

For now let us suppose that $V \approx V^{*}$, via a choice of inner product $\langle$,$\rangle ,$ so that $L$ is given by a pair of maps $\mathbf{a}, \mathbf{b}: \mathbb{R}^{n} \rightarrow V$ such that $\mathbf{a}^{*} \mathbf{b}$ is skew and $\operatorname{ker} \mathbf{a} \cap \operatorname{ker} \mathbf{b}=\{0\}$. We will see that $\mathbf{a}-\mathbf{b}$ and $\mathbf{a}+\mathbf{b}$ are invertible.

Suppose that $x \in \operatorname{ker} \mathbf{a}-\mathbf{b}$, so that $\mathbf{a} x=\mathbf{b} x$. Then $\left\langle\mathbf{a}^{*} \mathbf{b} x, x\right\rangle+\left\langle\mathbf{b}^{*} \mathbf{a} x, x\right\rangle=0$ implies $\left\langle\mathbf{a}^{*} \mathbf{a} x, x\right\rangle+\left\langle\mathbf{b}^{*} \mathbf{b} x, x\right\rangle=0$. But this says that $\|\mathbf{a} x\|^{2}+\|\mathbf{b} x\|^{2}=0$, and so $\mathbf{a} x=0$ and $\mathbf{b} x=0$. Therefore $x \in \operatorname{ker} \mathbf{a} \cap \operatorname{ker} \mathbf{b}$; thus $x=0$, and $\mathbf{a}-\mathbf{b}$ is invertible; similarly for $\mathbf{a}+\mathbf{b}$.

Now suppose that $V \approx \mathbb{R}^{n}$, and let us identify $\mathbb{R}^{n}$ with $\left(\mathbb{R}^{n}\right)^{*}$ via the canonical metric on $\mathbb{R}^{n}$. Finally let $\mathbf{e}_{i}$ be the $i$ th canonical basis element of $\mathbb{R}^{n}$. Then if we choose basis vectors $\mathbf{e}_{i} \oplus\{0\},\{0\} \oplus \mathbf{e}_{j}$ of $\mathbb{R}^{n} \oplus\left(\mathbb{R}^{n}\right)^{*}$, the form $\langle,\rangle_{+}$looks like

$$
\left(\begin{array}{ll}
0 & \mathbf{I} \\
\mathbf{I} & 0
\end{array}\right) \text {. }
$$

We may diagonalize this form by a change of basis. Explicitly we get

$$
\left(\begin{array}{cc}
\mathbf{I} & 0 \\
0 & -\mathbf{I}
\end{array}\right)
$$

in the basis given by

$$
\begin{aligned}
& y_{i}=\frac{\sqrt{2}}{2}\left(\mathbf{e}_{i} \oplus\{0\}+\{0\} \oplus \mathbf{e}_{i}\right), \\
& x_{i}=\frac{\sqrt{2}}{2}\left(\mathbf{e}_{i} \oplus\{0\}-\{0\} \oplus \mathbf{e}_{i}\right) .
\end{aligned}
$$

Thus the pairing $\langle,\rangle_{+}$has signature $(+1, \ldots,+1,-1, \ldots,-1)$, i.e., it has positive and negative definite subspaces in the dimension of $V$. Furthermore maximal isotropy occurs in the dimension of $V$.

Since a Dirac structure $L$ is isotropic under the pairing $\langle,\rangle_{+}$, it may not intersect any subspace of $\mathbb{R}^{n} \oplus\left(\mathbb{R}^{n}\right)^{*}$ which is definite under the pairing $\langle,\rangle_{+}$. Let us denote by $P$ the positive definite space spanned by the $y$ 's and by $N$ the negative definite space spanned by the $x$ 's. It follows that $L$ intersects both $N$ and $P$ transversally and thus may be realized as the graph of a linear map $A: N \rightarrow P$; therefore, we have $y=A x$.

Now the norm of $(x, y) \in N \oplus P$ under $\langle,\rangle_{+}$is zero, but because $x \in$ $N$ and $y \in P$ we have: $\langle(x, y),(x, y)\rangle_{+}=\|y\|-\|x\|$, and thus $\|y\|=$ $\|x\|$. Therefore the map $A: N \rightarrow P$ is norm preserving. Conversely, if we are provided with a norm preserving map $N \rightarrow P$, its graph is isotropic by definition, and is therefore a Dirac structure. Thus the set of Dirac structures on $\mathbb{R}^{n}$ is in one-to-one correspondence with the set of norm preserving maps $N \rightarrow P$.

Suppose that we have a basis representation $(\mathbf{a}, \mathbf{b})$ of a Dirac structure $L$. 
Then we may solve explicitly

$$
\begin{gathered}
(x, y)=\frac{\sqrt{2}}{2}((\mathbf{a}-\mathbf{b}) \mathbf{e},(\mathbf{a}+\mathbf{b}) \mathbf{e}) \in N \oplus P, \\
y=\frac{\sqrt{2}}{2}(\mathbf{a}+\mathbf{b}) \mathbf{e}, \\
x=\frac{\sqrt{2}}{2}(\mathbf{a}-\mathbf{b}) \mathbf{e} .
\end{gathered}
$$

Now by the discussion following the definition, we know that $\mathbf{a}-\mathbf{b}$ is invertible; thus we may solve

$$
\mathbf{e}=\frac{\sqrt{2}}{2}(\mathbf{a}-\mathbf{b})^{-1} x
$$

which with equation (1.2.7b) finally gives us a solution for the map $A: N \rightarrow P$, namely

$$
y=(\mathbf{a}+\mathbf{b})(\mathbf{a}-\mathbf{b})^{-1} x .
$$

This establishes the main facts we need to know about Dirac structures on $\mathbb{R}^{n}$, and so we return to the general case of Dirac structures on a vector space $V$.

1.3. Equivalence classes of basis representations. Let $(\mathbf{a}, \mathbf{b})$ be a basis representation of a Dirac structure on $\mathbb{R}^{n}$. We begin with a lemma:

Lemma 1.3.1. $\left(\mathbf{a}^{*}+\mathbf{b}^{*}\right)(\mathbf{a}+\mathbf{b})=\left(\mathbf{a}^{*}-\mathbf{b}^{*}\right)(\mathbf{a}-\mathbf{b})$.

Proof. Since $\mathbf{a}^{*} \mathbf{b}+\mathbf{b}^{*} \mathbf{a}=0$, we may add these terms to $\mathbf{a}^{*} \mathbf{a}+\mathbf{b}^{*} \mathbf{b}$ to get the left-hand side, or we may subtract them to get the right-hand side.

Proposition 1.3.2. Define $\mathbf{U}=(\mathbf{a}+\mathbf{b})(\mathbf{a}-\mathbf{b})^{-1}$. Then $\mathbf{U U}^{*}=\mathbf{I}$, i.e., $\mathbf{U}$ is orthogonal.

Proof. We compute $\mathbf{U U}^{*}$ :

$$
\begin{aligned}
(\mathbf{a}+\mathbf{b})(\mathbf{a}-\mathbf{b})^{-1}\left(\mathbf{a}^{*}-\mathbf{b}^{*}\right)^{-1}\left(\mathbf{a}^{*}+\mathbf{b}^{*}\right) & =(\mathbf{a}+\mathbf{b})\left[\left(\mathbf{a}^{*}-\mathbf{b}^{*}\right)(\mathbf{a}-\mathbf{b})\right]^{-1}\left(\mathbf{a}^{*}+\mathbf{b}^{*}\right) \\
& =(\mathbf{a}+\mathbf{b})\left[\left(\mathbf{a}^{*}+\mathbf{b}^{*}\right)(\mathbf{a}+\mathbf{b})\right]^{-1}\left(\mathbf{a}^{*}+\mathbf{b}^{*}\right) \\
& =\mathbf{I} . \quad
\end{aligned}
$$

Therefore $\mathbf{U}=(\mathbf{a}+\mathbf{b})(\mathbf{a}-\mathbf{b})^{-1}$ is orthogonal; the map $(a, b) \rightarrow U$ will be called the generalized Cayley transform. If $a$ is invertible, it becomes the Cayley transform

$$
(\mathbf{a}, \mathbf{a}) \rightarrow \mathbf{b a}^{-1} \rightarrow\left(\mathbf{I}+\mathbf{b a}^{-1}\right)\left(\mathbf{I}-\mathbf{b a}^{-1}\right)^{-1},
$$

since $\mathbf{b a}^{-1}$ is skew symmetric; a similar argument holds if $\mathbf{b}$ is invertible.

Let $(\mathbf{a}, \mathbf{b}) \approx(\underline{\mathbf{a}}, \underline{\mathbf{b}})$ denote the equivalence relation on pairs of maps that satisfy equations (1.2.1) and (1.2.2) and which are basis representations for the same Dirac structure. 
Theorem 1.3.3. The following are equivalent:

(1) $(\mathbf{a}, \mathbf{b}) \approx(\underline{\mathbf{a}}, \underline{\mathbf{b}})$.

(2) $(\mathbf{a}, \mathbf{b})=(\mathbf{a} \gamma, \mathbf{b} \gamma)$ for some $\gamma \in \mathrm{Gl}(n)$.

(3) $\mathbf{a}^{*} \underline{b}+\mathbf{b}^{*} \underline{a}=0$.

(4) $\mathbf{U}=\underline{\mathbf{U}}$, i.e., $(\mathbf{a}+\mathbf{b})(\mathbf{a}-\mathbf{b})^{-1}=(\underline{\mathbf{a}}+\underline{\mathbf{b}})(\underline{\mathbf{a}}-\underline{\mathbf{b}})^{-1}$.

Proof. (2) $\Rightarrow$ (3) We have $\underline{\mathbf{a}}=\mathbf{a} \gamma$ and $\underline{\mathbf{b}}=\mathbf{b} \gamma$, so that

$$
\mathbf{a}^{*} \underline{\mathbf{b}}+\mathbf{b}^{*} \underline{\mathbf{a}}=\left(\mathbf{a}^{*} \mathbf{b}+\mathbf{b}^{*} \mathbf{a}\right) \gamma=0,
$$

which is condition (3).

(3) $\Rightarrow(4) \mathbf{a}^{*} \underline{\mathbf{b}}+\mathbf{b}^{*} \underline{\mathbf{a}}=0$ implies that $\left(\mathbf{a}^{*}+\mathbf{b}^{*}\right)(\underline{\mathbf{a}}+\underline{\mathbf{b}})=\left(\mathbf{a}^{*}-\mathbf{b}^{*}\right)(\underline{\mathbf{a}}-\underline{\mathbf{b}})$ by the same reasoning as in the lemma above. Now we may multiply through by $(\underline{\mathbf{a}}-\underline{\mathbf{b}})^{-1}$ to get

$$
\left(\mathbf{a}^{*}+\mathbf{b}^{*}\right)(\underline{\mathbf{a}}+\underline{\mathbf{b}})(\underline{\mathbf{a}}-\underline{\mathbf{b}})^{-1}=\left(\mathbf{a}^{*}-\mathbf{b}^{*}\right)
$$

which implies $(\underline{\mathbf{a}}+\underline{\mathbf{b}})(\underline{\mathbf{a}}-\underline{\mathbf{b}})^{-1}=\left(\mathbf{a}^{*}+\mathbf{b}^{*}\right)^{-1}\left(\mathbf{a}^{*}-\mathbf{b}^{*}\right)$. So by definition we have $\underline{\mathbf{U}}=\left\{(\mathbf{a}-\mathbf{b})(\mathbf{a}+\mathbf{b})^{-1}\right\}^{*}=\left\{\mathbf{U}^{-1}\right\}^{*}=\mathbf{U}$. This establishes (4).

$(4) \Rightarrow(1)$. This follows from the fact that the Dirac structure induced by a basis representation is the graph of the generalized Cayley transformation.

$(1) \Rightarrow(2)$ Since the pair of maps $(\mathbf{a}, \mathbf{b})$ is determined by a choice of basis, it follows that if $(\mathbf{a}, \mathbf{b})$ and $(\underline{\mathbf{a}}, \underline{\mathbf{b}})$ have the same Dirac structure, then one may be obtained from the other by a change of basis in $L$. This is exactly statement (2).

Corollary. $(\mathbf{a}, \mathbf{b}) \approx(\mathbf{a} \gamma, \mathbf{b} \gamma)$ for all $\gamma \in \mathrm{Gl}(n)$.

Proof. Both representations have the same generalized Cayley transformation.

Theorem 1.3.3 shows that the action of $\operatorname{GL}(n)$ on $(\mathbf{a}, \mathbf{b})$ given by $(\mathbf{a}, \mathbf{b}) \times$ $\gamma=(\mathbf{a} \gamma, \mathbf{b} \gamma)$ amounts to a change of basis in our "reference space", and therefore $(\mathbf{a} \gamma, \mathbf{b} \gamma)$ still represents the same Dirac structure $L$. The theorem also shows that the map $(\mathbf{a}, \mathbf{b}) \rightarrow \mathbf{U}$ is invariant under this action. We also know that every $\mathbf{U}$ arises in the image of the ordinary Cayley transformation. Thus the space of Dirac structures on $V$ is in one-to-one correspondence with the group $\mathrm{O}(n)$. It follows that if $\mathbf{a}$ or $\mathbf{b}$ is invertible, which is the case in Examples 1.1.2 and 1.1.3, then it is possible by a change of basis to reduce it to the identity, i.e., we may find a change of basis so that $(\mathbf{a}, \mathbf{b})$ takes the form $(\mathbf{I}, \mathbf{b})$ or $(\mathbf{a}, \mathbf{I})$, respectively.

Now let $L$ be a Dirac structure on $V$ with basis representation $(\mathbf{a}, \mathbf{b})$. Then there is an action of $\mathrm{GL}(V)$ given by $(\mathbf{a}, \mathbf{b}) \times \delta=\left(\delta^{-1} \mathbf{a}, \delta^{*} \mathbf{b}\right)$ whose orbits are the isomorphism classes of Dirac structures on $V$. Suppose that for some choice of $\delta$ this leaves the Dirac structure invariant. Then by (3) of Theorem 1.3.3 we have

$$
\mathbf{a}^{*} \delta^{*} \mathbf{b}+\mathbf{b}^{*} \delta^{-1} \mathbf{a}=0 .
$$

Definition 1.3.4. If $\delta$ satisfies (1.3.2), we say that $\delta$ is a Dirac automorphism. 
In Example 1.1.2 we may change basis so that $(\mathbf{a}, \mathbf{b})$ takes the form $(\mathbf{a}, \mathbf{I})$, where $\mathbf{a}^{*}=-\mathbf{a}$, so that equation (1.2.1) takes the form $\mathbf{a}^{*} \delta^{*}+\delta^{-1} \mathbf{a}=0$, which may be rewritten as

$$
\delta \mathbf{a} \delta^{*}=\mathbf{a} .
$$

In Example 1.1.3 we may change coordinates so that $(\mathbf{a}, \mathbf{b})$ takes the form $(\mathbf{I}, \mathbf{b}), \mathbf{b}^{*}=-\mathbf{b}$, and reason as above to get

$$
\delta^{*} \mathbf{b} \delta=\mathbf{b} .
$$

Equations (1.3.3) and (1.3.4) are the automorphism equations for skew symmetric bilinear forms on $V^{*}$ and $V$ respectively.

1.4. Induced Dirac structures. We now see how a Dirac structure on $V$ is passed to a subspace $W \subset V$. Suppose that the structure on $V$ may be viewed as $E \subset V$ with a skew 2-form $\Omega_{E}: E \rightarrow E^{*}$. Then the inherited structure on $W$ is easy to see: it consists of the subspace $E \cap W$ of $W$ and the restriction to this subspace of the 2-form $\Omega_{E}$.

One obtains an equivalent picture using the formulation of a Dirac structure on $V$ as a subspace $L \subset V \oplus V^{*}$ which is maximally isotropic under the symmetric pairing $\langle,\rangle_{+}$. We will denote by $\circ$ the natural annihilator, and by $\perp$ the annihilator with respect to the symmetric pairing $\langle,\rangle_{+}$.

Consider the space $W \oplus V^{*}$ : we have $\left(W \oplus V^{*}\right)^{\perp}=\{0\} \oplus W^{\circ} \subset W \oplus V^{*}$, and therefore we may form the quotient space

$$
\frac{W \oplus V^{*}}{\left(W \oplus V^{*}\right)^{\perp}}=\frac{W \oplus V^{*}}{\{0\} \oplus W^{\circ}} \approx W \oplus W^{*} .
$$

Thus we get an exact sequence

$$
0 \rightarrow\{0\} \oplus W^{\circ} \stackrel{i}{\rightarrow} W \oplus V^{*} \stackrel{\pi}{\rightarrow} W \oplus W^{*} \rightarrow 0,
$$

with $i=$ inclusion and $\pi(v, \xi)=\left(v,\left.\xi\right|_{W}\right)$. The image of the Dirac structure $L$ on $V$ under this map will be called $L_{W}$. Consider now the second exact sequence and its inclusion in the first:

$$
\begin{gathered}
0 \rightarrow\{0\} \oplus W^{\circ} \rightarrow \underset{\uparrow}{W \oplus V^{*}} \rightarrow W \oplus W^{*} \rightarrow 0 \\
0 \rightarrow L \cap\left(\{0\} \oplus W^{\circ}\right) \rightarrow L \cap\left(W \oplus V^{*}\right) \rightarrow L_{W} \rightarrow 0
\end{gathered}
$$

Thus $L_{W}$ is defined to be the subspace of $W \oplus W^{*}$ which is the natural image of the projection $\pi$ as shown above. Note the natural isomorphism

$$
L_{W} \approx \frac{L \cap W \oplus V^{*}}{L \cap\{0\} \oplus W^{\circ}} .
$$

Now all of the above maps preserve the pairings $\langle,\rangle_{+}$and $\langle,\rangle_{-}$on $V \oplus V^{*}$ and $W \oplus W^{*}$. We wish to show that $\left.L_{W}\right)^{\perp}=L_{W}$ with respect to $\langle,\rangle_{+}$on $W \oplus W^{*}$. 
To see that $L_{W} \subset\left(L_{W}\right)^{\perp}$, first note that $L=L^{\perp}$; let $x \in L_{W}$. Then $\pi(a)=$ $x$ for some $a \in L \cap\left(W \oplus V^{*}\right)$. Now $a \in L=L^{\perp}$, so $a \in\left(L \cap\left(W \oplus V^{*}\right)\right)^{\perp}$; since $\pi$ preserves $\langle,\rangle_{+}$, we have $\pi(a) \in\left(L_{W}\right)^{\perp}$. Therefore $L_{W} \subset\left(L_{W}\right)^{\perp}$.

Now consider $\left(L_{W}\right)^{\perp} \subset W \oplus W^{*}$; let $\mu \in\left(L_{W}\right)^{\perp}$. Then $\pi^{\prime}(a)=\mu$ for some $a \in W \oplus V^{*}$, and because all the maps preserve $\langle,\rangle_{+}$, we have $a \in$ $\left(L \cap\left(W \oplus V^{*}\right)\right)^{\perp}$. Thus we have

$$
\begin{aligned}
a & \in\left(L \cap\left(W \oplus V^{*}\right)\right)^{\perp} \cap\left(W \oplus V^{*}\right) \\
& =\left(L^{\perp}+\left(W \oplus V^{*}\right)^{\perp}\right) \cap\left(W \oplus V^{*}\right) \\
& =\left(L_{+}\left(\{0\} \oplus W^{\circ}\right)\right) \cap\left(W \oplus V^{*}\right) \\
& =L \cap\left(W \oplus V^{*}\right)+\{0\} \oplus W^{\circ} .
\end{aligned}
$$

Therefore we may find $b \in L \cap\left(W \oplus V^{*}\right)$ and $c \in\{0\} \oplus W^{\circ}$ such that $a=b+c$; now commutativity of the diagram gives us $\pi(a)=\mu$ and $\pi(a)=\pi(b)+\pi(c)=$ $\pi(b)$. Therefore $\mu=\pi(b) \in L_{W}$. This shows that $\left(L_{W}\right)^{\perp} \subset L_{W}$. Therefore $L_{W}=\left(L_{W}\right)^{\perp}$ and therefore $L_{W}$ is a Dirac structure.

To verify that this is equivalent to the structure described in the first paragraph of this subsection, consider the commuting exact sequences:

$$
\begin{array}{cc}
0 \rightarrow\{0\} \oplus W^{\circ} \rightarrow W \oplus V^{*} \rightarrow W \oplus V^{*} \rightarrow 0 \\
\quad \downarrow \rho \quad \downarrow \rho & \downarrow \rho \\
0 \quad \rightarrow 0 & \rightarrow \rho(L) \cap W \rightarrow \rho\left(L_{W}\right) \rightarrow 0
\end{array}
$$

where $\rho$ is projection onto the first component. Clearly this shows that $\rho\left(L_{W}\right)$ $\approx \rho(L) \cap W$, so that the domain of the 2-form induced by $L_{W}$ is the intersection of $W$ with the domain of the 2-form induced by $L$. The 2-form on $\rho\left(L_{W}\right)$ satisfies $\rho^{*}(\Omega)=\langle,\rangle_{-}$, and therefore corresponds on $\rho(L) \cap W$ to the restriction of the 2-form on $\rho(L)$. Thus the two descriptions of Dirac reduction are the same.

Finally, consider the characteristic distribution $\operatorname{ker}(\Omega)=L_{W} \cap W$ (this may be viewed as a subspace of $L_{W}$ or of $W$ ). This corresponds to the kernel of $\langle,\rangle_{-}$:

Therefore

$$
\pi\left(\operatorname{ker}\langle,\rangle_{-}\right)=\pi\left(L \cap\left(W \oplus W^{\circ}\right)\right) \approx \frac{L \cap\left(W \oplus W^{\circ}\right)}{L \cap\left(\{0\} \oplus W^{\circ}\right)} .
$$

$$
L_{W} \cap W \approx \frac{L \cap\left(W \oplus W^{\circ}\right)}{L \cap\left(\{0\} \oplus W^{\circ}\right)} .
$$

\section{SMOoth Dirac STRUCTURES}

2.1. Lie algebroids. A Lie algebroid is a vector bundle $A$ over $P$ with the following additional structure:

(1) There is a Lie algebra bracket [, ] on sections of $A$.

(2) There is a bundle map $\rho: A \rightarrow T P$, called an anchor, for which the bracket on sections acts as a derivation, i.e., $[f \mu, \eta]=f[\mu, \eta]-$ $(\rho(\eta) \cdot f) \mu$ whenever $f \in C^{\infty}(P)$, and $\mu, \eta$ are sections of $A$. 
(3) The map $\rho$ is a Lie algebra homomorphism on sections.

(See Mackenzie [1987].)

Example 2.1.1. For any manifold $P$ the bundle $T P$ is a Lie algebroid with [ , ] the usual Jacobi-Lie bracket and $\rho: T P \rightarrow T P$ equal to the identity map.

Example 2.1.2. Let $P$ be a Poisson manifold. There is an algebroid bracket on $T^{*} P$, a bracket on 1 -forms, which for exact 1 -forms is given by $\{d f, d g\}=$ $d\{f, g\}$; the anchor map on exact 1 -forms if $\rho(d f)=-X_{f}$, the Hamiltonian vector field generated by $f$. We will see later how to write this algebroid bracket on all 1-forms (see Coste, Dazord, and Weinstein [1986]).

Note that condition (3) is equivalent to the Jacobi identity

$$
\{f,\{g, h\}\}+\{h,\{f, g\}\}+\{g,\{h, f\}\}=0 \text {. }
$$

Theorem 2.1.3. Let $A$ be a Lie algebroid over $P$ with anchor $\rho: E \rightarrow T P$. Then $\rho(A)$ is an integrable distribution (in the sense of Sussman [1973]).

Proof. Let $\mathbf{e}_{1}, \ldots, \mathbf{e}_{n}$ be a local basis of sections of $A$. Then we have:

$$
\left[\mathbf{e}_{i}, \mathbf{e}_{j}\right]=c_{i j}^{k} \mathbf{e}_{k} \quad \text { for some } c_{i j}^{k} \in C^{\infty}(P)
$$

Condition (3) tells us that

$$
\left[\rho\left(\mathbf{e}_{i}\right), \rho\left(\mathbf{e}_{j}\right)\right]=\rho\left(\left[\mathbf{e}_{i}, \mathbf{e}_{j}\right]\right)=\rho\left(c_{i j}^{k} \mathbf{e}_{k}\right)=c_{i j}^{k} \rho\left(\mathbf{e}_{k}\right) .
$$

This is the integrability condition of Sussman.

(For a discussion of singular foliations and their integral submanifolds see Dazord [1985], and Sussman [1973].)

Let $A$ be a Lie algebroid. We will see that the dual bundle $A^{*}$ to a Lie algebroid $A$ inherits a Poisson structure, i.e., a Poisson algebra on $C^{\infty}\left(A^{*}\right)$, such that brackets of linear functions are again linear; this is a natural extension of the Lie-Poisson structure on the dual of a Lie algebra.

Let $\mu, \eta$ be sections of $A$, let $f$ be a function on $P$, and let $\pi$ be the bundle projection of $A^{*}$ onto $P$. Then $\mu$ and $\eta$ determine linear functions on $A^{*}$ which we will denote by $\tilde{\mu}$ and $\tilde{\eta} ; f \circ \pi$ is a function on $A^{*}$ which is constant on each fiber.

We now show that there is a unique Poisson structure on $A^{*}$ satisfying:
(a) $\{\tilde{\mu}, \tilde{\eta}\}=[\widetilde{\mu, \eta}]$.
(b) $\{\tilde{\mu}, f \circ \pi\}=(\rho(\mu) \cdot f) \circ \pi$.
(c) $\{f \circ \pi, g \circ \pi\}=0$. 
The only nontrivial part of the Jacobi identity mixes brackets in (a) and (b):

$$
\begin{aligned}
&\{\{\tilde{\mu}, \tilde{\eta}\}, f \circ \pi\}+\{\{\tilde{\eta}, f \circ \pi\}, \tilde{\mu}\}+\{\{f \circ \pi, \tilde{\mu}\}, \tilde{\eta}\} \\
&=\{[\tilde{\mu, \eta}], f \circ \pi\}+\{\rho(\eta) \cdot f) \circ \pi, \tilde{\mu}\}-\{(\rho(\mu) \circ f) \circ \pi, \tilde{\eta}\} \\
&=(\rho([\mu, \eta]) \cdot f) \circ \pi-(\rho(\mu) \cdot(\rho(\eta) \cdot f)) \circ \pi+(\rho(\eta) \cdot(\rho(\mu) \cdot f)) \circ \pi \\
&=(\rho([\mu, \eta]) \cdot f) \circ \pi-([\rho(\mu), \rho(\eta)] \cdot f) \circ \pi \\
&=((\rho([\mu, \eta]-[\rho(\mu), \rho(\eta)]) \cdot f) \circ \pi \\
&=0 \quad(\text { since } \rho \text { is a homomorphism on sections }) .
\end{aligned}
$$

It will be useful to continue in local coordinates: choose a local basis of sections $\mathbf{e}_{i}$ of $A$ and a system of local coordinates $x^{i}$. Then these induce coordinates $\left(x^{i}, \mu_{i}\right)$ on $A^{*}$ such that $\mu_{i}=\tilde{\mathbf{e}}_{i}=\left\langle\cdot \mid \mathbf{e}_{i}\right\rangle$ (these are linear coordinates on the bundle $A^{*}$ ). In these coordinates we define the structure functions and components of the anchor map:

$$
\left[\mathbf{e}_{i}, \mathbf{e}_{j}\right]=c_{i j}^{k} \mathbf{e}_{k} \quad \text { and } \quad \rho\left(\mathbf{e}_{i}\right)=\rho_{i}^{j}(x) \frac{\partial}{\partial x^{j}} .
$$

Then equations (a), (b), (c) determine the brackets of the coordinate functions:

$$
\left\{\mu_{i}, \mu_{j}\right\}=c_{i j}^{k} \mu_{k}, \quad\left\{\mu_{i}, x^{j}\right\}=\rho_{i}^{j}, \quad\left\{x^{i}, \mu_{j}\right\}=-\rho_{i}^{j}, \quad\left\{x^{i}, x^{j}\right\}=0 .
$$

Then brackets determined by these equations satisfy the Jacobi identity, and since a Poisson structure is determined by its values on coordinate functions, it follows that conditions (a), (b), (c) determine a Poisson structure on $A^{*}$. Thus we have shown that $A^{*}$ has a Poisson structure in which the bracket of linear functions is again linear.

We now discuss a converse. Suppose we have a Poisson structure $\{$,$\} on a$ bundle $A^{*}$ over $P$, such that the bracket of linear functions is again linear (in this discussion, $A^{*}$ will be the arbitrary vector bundle, and $A$ will be its dual).

Let $\mu, \eta \in \Gamma(A)$ so that $\tilde{\mu}, \tilde{\eta} \in C^{\infty}\left(A^{*}\right)$. Define

$$
[\mu, \eta]=\{\tilde{\mu}, \tilde{\eta}\} \text {. }
$$

The fact that the Poisson bracket of linear functions is again linear implies that $[\mu, \eta]$ is a section of $A$, i.e., an element of $\Gamma(A)$. Furthermore this bracket satisfies the Jacobi identity since the Poisson bracket does. Therefore the vector bundle $A$ has a Lie algebra bracket on sections. We shall establish an anchor map $\rho$ and a derivation law for this bracket, thereby showing that $A$ is a Lie algebroid.

Let $f$ be a function on $P$, and let $\mu$ be a linear function on $A^{*}$; then $f$ may be viewed as a function on $A^{*}$ which is constant on fibers. The derivation law for the Poisson bracket states that $\{\mu, f \eta\}=f\{\mu, \eta\}+\eta\{\mu, f\}$; of these three terms, the first two are linear functions, and therefore $\{\mu, f\}$ is a function on $A^{*}$ which is constant on fibers. Another application of the Leibniz identity $\{\mu, f g\}=g\{\mu, f\}+f\{\mu, g\}$ tells us that $\mu$ determines a vector field $\rho(\mu)$ on $P$ by the relation $\rho(\mu) \cdot f=\{\mu, f\}$. To establish that $\rho$ is an anchor map, it 
remains to show that the map $\mu \rightarrow \rho(\mu)$ is induced by a bundle map. Consider the Leibniz identity again:

$$
\{f \mu, g\}=f\{\mu, g\}+\mu\{f, g\} .
$$

The first two terms are constant on fibers, and $\mu$ is any linear function, so we must have

$$
\{f, g\}=0 \text {. }
$$

Thus,

$$
\{f \mu, g\}=f\{\mu, g\}
$$

and therefore

$$
\rho(f \mu)=f \rho(\mu) .
$$

This shows that $\rho$ is a bundle map, and is therefore an anchor map. Finally, we have

$$
[\mu, f \eta]=\{\mu, f \eta\}=f\{\mu, \eta\}+\eta\{\mu, f\}=f\{\mu, \eta\}+(\rho(\mu) \cdot f) \eta
$$

which establishes the derivation law for the Lie bracket on sections of $A$. This shows that $A$ is a Lie algebroid, whose bracket [, ] and anchor map $\rho$ satisfy conditions (a), (b), (c), and therefore the Poisson structure on $A^{*}$ arises as the dual to the algebroid $A$.

Thus we have shown:

Theorem 2.1.4. The dual bundle to a Lie algebroid is a Poisson manifold such that the Poisson bracket of linear functions is again linear.

Furthermore any vector bundle with such a Poisson structure is a dual bundle to a Lie algebroid, and its Poisson structure is inherited as such.

2.2. Dirac structures on manifolds. In $\S 1$ we saw that we could think of a Dirac structure on a vector space $V$ as a subspace $L \subset V \oplus V^{*}$ which is isotropic under $\langle,\rangle_{+}$. We now wish to extend some of the results of the linear case to manifolds $P$. We may define natural symmetric and skew-symmetric pairings on $T P \oplus T^{*} P$ :

$$
\begin{aligned}
& \langle(X, \omega),(Y, \mu)\rangle_{+}=\frac{1}{2}(\omega(Y)+\mu(X)), \\
& \langle(X, \omega),(Y, \mu)\rangle_{-}=\frac{1}{2}(\omega(Y)-\mu(X)) .
\end{aligned}
$$

Definition 2.2.1. An almost-Dirac structure, or a Dirac bundle, on a manifold $P$ is a subbundle $L \subset T P \oplus T^{*} P$ which is maximally isotropic under the symmetric pairing $\langle,\rangle_{+}$.

We should add that a Dirac structure will be defined as an almost-Dirac structure satisfying a certain integrability condition; later this will be called an integrable Dirac structure. 
Applying to each fiber of $L$ the characteristic equations, we get the characteristic equations of a Dirac bundle:

$$
\begin{aligned}
& \rho(L)^{\circ}=L \cap T^{*} P, \\
& \rho^{*}(L)=(L \cap T P)^{\circ} .
\end{aligned}
$$

As in the linear case, we get a 2-form, but now it is on the description $\rho(L)$ :

$$
\Omega_{L}: \rho(L) \rightarrow \rho(L)^{*}
$$

and

$$
L \cap T P=\operatorname{ker} \Omega_{L}
$$

These are also pointwise equations.

Example 2.2.2. Let $B: T^{*} P \rightarrow T P$ define a Poisson structure on $P$, and let

$$
L=\operatorname{graph}(B) \subset T P \oplus T^{*} P .
$$

Thus the distribution $\rho(L)$ equals $\operatorname{Im}(B)$, which is an integrable singular distribution. Therefore Poisson manifolds have symplectic leaves, even at singular points; indeed, we have $\rho^{*}(L)=T^{*} P$ so that $L \cap T P=0$ by $(2.2 .4)$, and therefore the 2-form $\Omega_{L}$ on the distribution $\rho(L)$ is nonsingular at each point by (2.2.6).

Example 2.2.3. Let $\Omega: T P \rightarrow T^{*} P$ be a closed 2-form, and let $L=\operatorname{graph} \Omega$; then $L \cap T P=\operatorname{ker} \Omega$ and $\rho(L)=T P$, so there is only one "leaf", namely $P$.

In these two examples there is the additional structure of a Jacobi identity:

$$
\begin{aligned}
{[B, B] } & =0, \\
d \Omega & =0 .
\end{aligned}
$$

In this section we will determine a condition, namely the vanishing of a 3-tensor on $L$, which will establish two things:

1. the integrability of $\rho(L)$ as a singular distribution;

2. closedness of the 2-form on each leaf of this distribution. Furthermore, in Example 2.2.2 or 2.2.3, the 3-tensor is $[B, B]$ or $d \Omega$ respectively.

2.3. Integrability of Dirac structures. We define a bilinear bracket operation on sections of $T P \oplus T^{*} P$ by

$$
[(X, \omega),(Y, \mu)]=\left([X, Y], \mathfrak{L}_{X} \mu-\mathfrak{L}_{Y} \omega+d\left(\langle(X, \omega),(Y, \mu)\rangle_{-}\right)\right) .
$$

In general, this is not a Lie-algebra bracket. If we restrict it to sections of $L$ we get

$$
[(X, \omega),(Y, \mu)]=\left([X, Y], \mathfrak{L}_{X} \mu-\mathfrak{L}_{Y} \omega+d(\omega(Y))\right) .
$$

Definition 2.3.1. If $\Gamma(L)$ is closed under this bracket, we call $L$ an integrable Dirac bundle.

We will see that $\left(L,\left.\rho\right|_{L},[],\right)$ is a Lie algebroid when $\Gamma(L)$ is closed under $[$,$] .$ 
Definition 2.3.2. We define $\mathbf{T}_{L}\left(\mathbf{e}_{1} \otimes \mathbf{e}_{2} \otimes \mathbf{e}_{3}\right)=\left\langle\left[\mathbf{e}_{1}, \mathbf{e}_{2}\right], \mathbf{e}_{3}\right\rangle_{+}$, where $\mathbf{e}_{i}$ are sections of $L$.

Proposition 2.3.3. $L$ is an integrable Dirac bundle if and only if $\mathbf{T}_{L}=0$.

Proof. Use the fact that $L$ is maximally isotropic under the bracket $\langle,\rangle_{+}$.

Now we compute

$$
\begin{aligned}
\mathbf{T}_{L}((X, \omega) \otimes(Y, \mu) \otimes(Z, \nu)) \\
=\left\langle\left([X, Y], \mathfrak{L}_{X} \mu-\mathfrak{L}_{Y} \omega+d(\omega(Y))\right),(Z, \nu)\right\rangle_{+} \\
=\frac{1}{2}\left\{\nu \cdot[X, Y]+\left(\mathfrak{L}_{X} \mu\right)(Z)-\left(\mathfrak{L}_{Y} \omega\right)(Z)+Z \cdot(\omega(Y))\right\} \\
=\frac{1}{2}\left\{\nu \cdot[X, Y]+\mathfrak{L}_{X}(\mu(Z))-\mu \cdot[X, Z]-\mathfrak{L}_{Y}(\omega(Z))\right. \\
\quad+\omega \cdot[Y, Z]+Z \cdot(\omega(Y))\} \\
=\frac{1}{2}\{\omega \cdot[Y, Z]+\mu \cdot[Z, X]+\nu \cdot[X, Y]+X \cdot \mu(Z) \\
\quad+Y \cdot \nu(X)+Z \cdot(\omega(Y))\} .
\end{aligned}
$$

Now we use the identity $d \omega(Y, Z)=Y \cdot \omega(Z)-Z \cdot \omega(Y)-\omega \cdot[Y, Z]$ in the form

$$
\omega \cdot[Y, Z]+Z \cdot \omega(Y)=Y \cdot \omega(Z)-d \omega(Y, Z) .
$$

Using the same formula for $\mu$ and $\nu$, and summing gives a useful alternate formula for $\mathbf{T}_{L}$, namely

$$
\begin{aligned}
\mathrm{T}_{L}((X, \omega) \otimes(Y, \mu) \otimes(Z, \nu)) \\
=\frac{1}{2}(X \cdot \mu(Z)+Y \cdot \nu(Y)+Z \cdot \omega(Y) \\
\quad+d \omega(Y, Z)+d \mu(Z, X)+d \nu(X, Y)) .
\end{aligned}
$$

This is the restriction to $L$ of the following totally skew form on $\Gamma\left(T P \oplus T^{*} P\right)$ :

$$
\begin{aligned}
& \mathbf{T}((X, \omega) \otimes(Y, \mu) \otimes(Z, \nu)) \\
&=-\left\{d \omega(Y, Z)+d \mu(Z, X)+d \nu(X, Y)+X \cdot\langle(Y, \mu),(Z, \nu)\rangle_{-}\right. \\
&\left.\quad+Y \cdot\langle(Z, \nu),(X, \omega)\rangle_{-}+Z \cdot\langle(X, \omega),(Y, \mu)\rangle_{-}\right\} .
\end{aligned}
$$

We will now see that $\mathbf{T}_{L}$ is a tensor. First notice that $\mathbf{T}_{L}$ is linear in $\mathbf{e}_{3}$ according to Definition 2.3.2. Since $\mathbf{T}_{L}$ is the restriction to $L$ of a totally skew symmetric form, it follows that $\mathbf{T}_{L}$ is linear in each of its arguments. Therefore $\mathbf{T}_{L}$ is a 3-tensor. Thus integrability of a Dirac structure is determined by the vanishing of a 3-tensor on $L$.

We now test the derivation property of this bracket:

$\left[\left(X_{1}, \omega_{1}\right),\left(X_{2}, \omega_{2}\right)\right]=\left(\left[X_{1}, X_{2}\right], \mathfrak{L}_{X_{1}} \omega_{2}-\mathfrak{L}_{X_{2}} \omega_{1}+d\left(\left\langle\left(X_{1}, \omega_{1}\right),\left(X_{2}, \omega_{2}\right)\right\rangle_{-}\right)\right)$, so we see that

$$
\begin{aligned}
& {\left[\left(f X_{1}, f \omega_{1}\right),\left(X_{2}, \omega_{2}\right)\right]} \\
& \quad=\left(\left[f X_{1}, X_{2}\right], \mathfrak{L}_{f X_{1}} \omega_{2}-\mathfrak{L}_{X_{2}} f \omega_{1}+d\left(f\left\langle\left(X_{1}, \omega_{1}\right),\left(X_{2}, \omega_{2}\right)\right\rangle_{-}\right)\right) .
\end{aligned}
$$


Now

$$
\begin{aligned}
\mathfrak{L}_{f X_{1}} \omega_{2}- & \mathfrak{L}_{X_{2}} f \omega_{1}+d\left(f\left\langle\left(X_{1}, \omega_{1}\right),\left(X_{2}, \omega_{2}\right)\right\rangle_{-}\right) \\
= & f \mathfrak{L}_{X_{1}} \omega_{2}+\omega_{2}\left(X_{1}\right) d f-f \mathfrak{L}_{X_{2}} \omega_{1}-\left(X_{2} \cdot f\right) \omega_{1} \\
& +d f\left\langle\mathbf{e}_{1}, \mathbf{e}_{2}\right\rangle_{-}+f d\left(\left\langle\mathbf{e}_{1}, \mathbf{e}_{2}\right\rangle_{-}\right) \\
= & f\left(\mathfrak{L}_{X_{1}} \omega_{2}-\mathfrak{L}_{X_{2}} \omega_{1}+d\left(\left\langle\mathbf{e}_{1}, \mathbf{e}_{2}\right\rangle_{-}\right)\right) \\
& +\omega_{2}\left(X_{1}\right) d f-\left(X_{2} \cdot f\right) \omega_{1}+d f\left\langle\mathbf{e}_{1}, \mathbf{e}_{2}\right\rangle_{-} \\
= & f \rho^{*}\left(\left[\mathbf{e}_{1}, \mathbf{e}_{2}\right]\right)-\left(X_{2} \cdot f\right) \omega_{1}+\omega_{2}\left(X_{1}\right) d f+\left\langle\mathbf{e}_{1}, \mathbf{e}_{2}\right\rangle_{-} d f .
\end{aligned}
$$

Finally, $\omega_{2}\left(X_{1}\right)+\left\langle\mathbf{e}_{1}, \mathbf{e}_{2}\right\rangle_{-}=\left\langle\mathbf{e}_{1}, \mathbf{e}_{2}\right\rangle_{+}$, and so

$$
\rho^{*}\left(\left[f \mathbf{e}_{1}, \mathbf{e}_{2}\right]\right)=f \rho^{*}\left(\left[\mathbf{e}_{1}, \mathbf{e}_{2}\right]\right)-\left(X_{2} \cdot f\right) \omega_{1}+\left\langle\mathbf{e}_{1}, \mathbf{e}_{2}\right\rangle_{+} d f .
$$

Also $\rho\left(\left[f \mathbf{e}_{1}, \mathbf{e}_{2}\right]\right)=f \rho\left(\left[\mathbf{e}_{1}, \mathbf{e}_{2}\right]\right)-\left(X_{2} \cdot f\right) X_{1}$.

If $L$ is isotropic under the pairing $\langle,\rangle_{+}$, this may be written as

$$
\begin{aligned}
\rho^{*}\left(\left[f \mathbf{e}_{1}, \mathbf{e}_{2}\right]\right) & =f \rho^{*}\left(\left[\mathbf{e}_{1}, \mathbf{e}_{2}\right]\right)-\left(\rho\left(\mathbf{e}_{2}\right) \cdot f\right) \rho^{*}\left(\mathbf{e}_{1}\right), \\
\rho\left(\left[f \mathbf{e}_{1}, \mathbf{e}_{2}\right]\right) & =f \rho\left(\left[\mathbf{e}_{1}, \mathbf{e}_{2}\right]\right)-\left(\rho\left(\mathbf{e}_{2}\right) \cdot f\right) \rho\left(\mathbf{e}_{1}\right),
\end{aligned}
$$

which is part of the condition for $L$ to be an algebroid.

Now for the Jacobi identity. Assuming that $\mathbf{T}=0$ we have

$$
\left[\left(X_{1}, \omega_{1}\right),\left(X_{2}, \omega_{2}\right)\right]=\left(\left[X_{1}, X_{2}\right], \mathfrak{L}_{X_{1}} \omega_{2}-\mathfrak{L}_{X_{2}} \omega_{2}+d \omega_{1}\left(X_{2}\right)\right)
$$

so the triple bracket will be

$$
\begin{aligned}
{\left[\left[\left(X_{1}, \omega_{1}\right),\left(X_{2}, \omega_{2}\right)\right],\left(X_{3}, \omega_{3}\right)\right] } & \\
= & \left(\left[\left[X_{1}, X_{2}\right], X_{3}\right],-\left(\mathfrak{L}_{X_{3}}\left(\mathfrak{L}_{X_{1}} \omega_{2}-\mathfrak{L}_{X_{2}} \omega_{2}\right)+d\left\{X_{3} \cdot \omega_{1}\left(X_{2}\right)\right\}\right.\right. \\
& \left.\left.-\mathfrak{L}_{\left[X_{1}, X_{2}\right]} \omega_{3}+d\left\{\omega_{3}\left(\left[X_{1}, X_{2}\right]\right)\right\}\right)\right) .
\end{aligned}
$$

Therefore, (minus) the second component of the right-hand side of the Jacobi identity is

$$
\begin{aligned}
\mathfrak{L}_{X_{3}} & \left(\mathfrak{L}_{X_{1}} \omega_{2}-\mathfrak{L}_{X_{2}} \omega_{1}\right)+d\left\{X_{3} \cdot \omega_{1}\left(X_{2}\right)\right\}-\mathfrak{L}_{\left[X_{1}, X_{2}\right]} \omega_{3}+d\left\{\omega_{3}\left(\left[X_{1}, X_{2}\right]\right)\right\} \\
& +\mathfrak{L}_{X_{1}}\left(\mathfrak{L}_{X_{2}} \omega_{3}-\mathfrak{L}_{X_{3}} \omega_{2}\right)+d\left\{X_{1} \cdot \omega_{2}\left(X_{3}\right)\right\}-\mathfrak{L}_{\left[X_{2}, X_{3}\right]} \omega_{1}+d\left\{\omega_{1}\left(\left[X_{2}, X_{3}\right]\right)\right\} \\
& +\mathfrak{L}_{X_{2}}\left(\mathfrak{L}_{X_{3}} \omega_{1}-\mathfrak{L}_{X_{1}} \omega_{3}\right)+d\left\{X_{2} \cdot \omega_{3}\left(X_{1}\right)\right\}-\mathfrak{L}_{\left[X_{3}, X_{1}\right]} \omega_{2}+d\left\{\omega_{2}\left(\left[X_{3}, X_{1}\right]\right)\right\} \\
= & \mathfrak{L}_{X_{3}} \mathfrak{L}_{X_{1}} \omega_{2}-\mathfrak{L}_{X_{1}} \mathfrak{L}_{X_{3}} \omega_{2}+\mathfrak{L}_{X_{1}} \mathfrak{L}_{X_{2}} \omega_{3}-\mathfrak{L}_{X_{2}} \mathfrak{L}_{X_{1}} \omega_{3}+\mathfrak{L}_{X_{2}} \mathfrak{L}_{X_{3}} \omega_{1} \\
& -\mathfrak{L}_{X_{3}} \mathfrak{L}_{X_{2}} \omega-\mathfrak{L}_{\left[X_{1}, X_{2}\right]} \omega_{3}-\mathfrak{L}_{\left[X_{2}, X_{3}\right]} \omega_{1}-\mathfrak{L}_{\left[X_{3}, X_{1}\right]} \omega_{2} \\
& +d\left\{X_{3} \cdot \omega_{1}\left(X_{2}\right)\right\}+d\left\{X_{1} \cdot \omega_{2}\left(X_{3}\right)\right\}+d\left\{X_{2} \cdot \omega_{3}\left(X_{1}\right)\right\} \\
& +d\left\{\omega_{3}\left(\left[X_{1}, X_{2}\right]\right)\right\}+\left\{\omega_{1}\left(\left[X_{2}, X_{3}\right]\right)\right\}+d\left\{\omega_{2}\left(\left[X_{3}, X_{1}\right]\right)\right\} \\
= & d\left\{X_{3} \cdot \omega_{1}\left(X_{2}\right)+\omega_{1}\left(\left[X_{2}, X_{3}\right]\right)\right\} \\
& +d\left\{X_{1} \cdot \omega_{2}\left(X_{3}\right)+\omega_{2}\left(\left[X_{3}, X_{1}\right]\right)\right\}+d\left\{X_{2} \cdot \omega_{3}\left(X_{1}\right)\right. \\
& \left.+\omega_{3}\left(\left[X_{1}, X_{2}\right]\right)\right\} \\
= & d\left\{\mathbf{T}\left(\left(X_{1}, \omega_{1}\right) \otimes\left(X_{2}, \omega_{2}\right) \otimes\left(X_{3}, \omega_{3}\right)\right)\right\} \\
= & 0 .
\end{aligned}
$$


Let us summarize what we have seen so far: the bracket and projection given above turn isotropic subbundles of $T P \oplus T^{*} P$ into Lie algebroids. Thus we have shown:

Theorem 2.3.4. An almost-Dirac structure $L$ is integrable if and only if $\left(L,\left.\rho\right|_{L},[],\right)$ is a Lie algebroid.

Therefore if $\mathbf{T}_{L}=0$, Theorem 2.1.3 implies that $\rho(L)$ is an integrable singular distribution, i.e., it has leaves $\Delta$ such that $T_{X} \Delta=\rho_{X}(L)$.

Corollary. If $L$ is an integrable Dirac bundle over $P$, then $\rho(L)$ generates a singular foliation of $P$. by

As in the linear case we may define a 2-form $\Omega_{L}: \rho(L) \rightarrow \rho(L)^{*}$ on $\rho(L)$

$$
\Omega_{L}(X) \cdot(Y)=\omega(Y) \quad \text { whenever }(X, \omega),(Y, \mu) \in L .
$$

$\Omega_{L}$ is a map $\Omega_{L}: T \Delta \rightarrow T^{*} \Delta$, i.e., $\Omega_{L}$ is a 2-form on each leaf $\Delta$.

Theorem 2.3.5. Let $\rho_{L}=i_{L_{\rho}}^{*}$, where $i_{L}: L \rightarrow T P \oplus T^{*} P$. Then

$$
\rho_{L}^{*} \Omega_{L}=i_{L}^{*}\left(\langle,\rangle_{-}\right) \text {. }
$$

Proof. This is the definition of $\Omega_{L}$.

Equation (2.3.3) shows that $\Omega_{L}$ is a smooth 2-form. We will now compute $\rho_{L}^{*} d \Omega_{L}$, a smooth skew symmetric 3 -tensor on the vector bundle $L$ :

$$
\begin{aligned}
\rho_{L}^{*} d \Omega_{L} & \left(\mathbf{e}_{1}, \mathbf{e}_{2}, \mathbf{e}_{3}\right)=d \Omega_{L}(X, Y, Z) \\
= & X \cdot \Omega_{L}(Y, Z)+Y \cdot \Omega_{L}(Z, X)+Z \cdot \Omega_{L}(X, Y) \\
& +\Omega_{L}(X,[Y, Z])+\Omega_{L}(Y,[Z, X])+\Omega_{L}(Z,[X, Y]) \\
= & -\mathbf{T}_{L}\left(\mathbf{e}_{1}, \mathbf{e}_{2}, \mathbf{e}_{3}\right) .
\end{aligned}
$$

Thus we have $\rho_{L}^{*} d \Omega_{L}=\mathbf{T}_{L}$.

Theorem 2.3.6. An integrable Dirac structure has a foliation by presymplectic leaves.

Proof. $\mathbf{T}_{L}=0$ implies that $d \Omega_{L}=0$, since $\rho_{L}$ is a surjection.

We now return to the examples of $\S 2.1$. Let $P$ be a Poisson manifold. We define a bracket on sections of $T^{*} P$. Let $\omega, \mu \in \Gamma\left(T^{*} P\right)$ and write $X_{\omega}=B(\omega)$ and $X_{\mu}=B(\mu)$, where $B: T^{*} P \rightarrow T P$ is the Poisson bundle map. Then we define

$$
\begin{aligned}
{[\omega, \mu] } & =\mathfrak{L}_{X_{\omega}} \mu-\mathfrak{L}_{X_{\mu}} \omega+d\left(\omega\left(X_{\mu}\right)\right) \\
& \left.\left.=X_{\omega}\right\rfloor d \mu-X_{\mu}\right\rfloor d \omega-d\left(\omega\left(X_{\mu}\right)\right) .
\end{aligned}
$$

(Note that the apparent asymmetry in the last term is not really an asymmetry since $\omega\left(X_{\mu}\right)+\mu\left(X_{\omega}\right)=0$.) Then we have

$$
\begin{aligned}
{[\omega, f \mu] } & \left.\left.=X_{\omega}\right\rfloor d(f \mu)-f X_{\mu}\right\rfloor d \omega-d\left(\omega\left(f X_{\mu}\right)\right) \\
& =f[\omega, \mu]+\left(X_{\omega} \cdot f\right) \mu-\mu\left(X_{\omega}\right) d f-\omega\left(X_{\mu}\right) d f \\
& =f[\omega, \mu]+\left(X_{\omega} \cdot f\right) \mu .
\end{aligned}
$$


Theorem 2.3.4 implies that this bracket satisfies the Jacobi identity (since $B: T^{*} P \rightarrow T P$ defines a Poisson structure). Therefore (2.3.4) makes $T^{*} P$ into a Lie algebroid.

Now let $L$ be the graph of $B: T^{*} P \rightarrow T P$. We know that $\rho^{*}: L \rightarrow T^{*} P$ is an isomorphism, so that: $L^{*} \approx T P$.

By Theorem 2.1.4 we see that $L^{*}$ inherits a Poisson structure. In the notation of $\S 2.1$, we have

$$
\mathbf{e}^{i}=d x^{i} \text { and } \mu^{j}=v^{j} .
$$

Let $\left\{x^{i}, x^{j}\right\}=\pi^{i j}$. Then we may solve for the structure functions of the Lie algebroid; all we need is the algebroid bracket on functions:

$$
\left\{d x^{i}, d x^{j}\right\}=d\left\{x^{i}, x^{j}\right\}=d \pi^{i j}=\pi^{i j},{ }_{k} d x^{k} .
$$

Therefore we have

$$
c_{k}^{i j}=\pi^{i j},{ }_{k} .
$$

We also have $\rho\left(\mathbf{e}^{i}\right)=\rho\left(d x^{i}\right)=\xi_{x^{i}}$, so that

$$
\rho_{i}^{j}=\rho\left(\mathbf{e}^{i}\right) \cdot x^{j}=\left\{x^{i}, x^{j}\right\}=\pi^{i j} .
$$

So equations (2.1.4) of $\S 2.1$ take the form

$$
\left\{v^{i}, v^{j}\right\}=\pi_{, k}^{i j} v^{k}, \quad\left\{x^{i}, v^{j}\right\}=\pi^{i j}, \quad\left\{v^{i}, x^{j}\right\}=-\pi^{i j}, \quad\left\{x^{i}, x^{j}\right\}=0 .
$$

This is the tangent Poisson bracket defined in Alvarez-Sanchez [1986].

Now we consider the case where $L$ is the graph of a presymplectic structure. In this case $\rho: L \rightarrow T P$ is an isomorphism, so that

$$
L^{*} \approx T^{*} P \text {. }
$$

It seems natural to choose canonical coordinates $q^{i}, p_{j}$ on $T^{*} P$. Then we have

$$
\mathbf{e}_{i}=\partial / \partial q^{i} \text { and } \mu^{j}=d q^{i}
$$

Therefore the structure functions are identically zero. As for the anchor map, we have

$$
\rho_{i}^{j}=\rho\left(\mathbf{e}^{i}\right) \cdot q^{j}=\delta_{i}^{j},
$$

so the bracket equations take the form

$$
\left\{p_{i}, p_{j}\right\}=0, \quad\left\{p_{i}, q^{j}\right\}=-\delta_{i}^{j}, \quad\left\{q^{i}, q^{j}\right\}=0 .
$$

These are the canonical Poisson bracket equations on the cotangent bundle of $P$.

2.4. Invariance under flows. Let $(X, \omega),(Y, \mu)$, and $(Z, \nu)$ be sections of an integrable Dirac structure $L$. Define

$$
X \cdot(Y, \mu) \equiv\left([X, Y], \mathfrak{L}_{X} \mu\right) .
$$


This is the infinitesimal analog of the action of $\mathrm{Gl}(V)$ in $\S 1$.

We know that $\left([X, Y], \mathfrak{L}_{X} \mu-\mathfrak{L}_{Y} \omega+d(\omega(Y))\right)$ is again in $L$, and thus annihilates $L$ under $\langle,\rangle_{+}$. Thus we have

$$
\begin{aligned}
{[(X, \omega),(Y, \mu)] } & =\left([X, Y], \mathfrak{L}_{X} \mu-\mathfrak{L}_{Y} \omega+d(\omega(Y))\right) \\
& \left.=\left([X, Y], \mathfrak{L}_{X} \mu\right)-(0, Y\rfloor d \omega\right) \\
& =X \cdot(Y, \mu)-(0, Y\rfloor d \omega) .
\end{aligned}
$$

Therefore

$$
\left.\langle X \cdot(Y, \mu),(Z, \nu)\rangle_{+}=\langle(0, Y\rfloor d \omega),(Z, \nu)\right\rangle_{+}=d \omega(Y, Z) .
$$

So

$X \cdot L \subset L \quad$ if and only if $\left.\quad d \omega\right|_{\rho(L)}=0$.

Thus we have shown:

Theorem 2.4.1. An integrable Dirac structure on $P$ is locally invariant under $X \in \rho(\Gamma(L))$ if about each point there is a function $H$ such that $(X, d H)$ is a local section of $L$.

Consider now the 2-form $\Omega_{L}$ on a leaf. By definition, we may consider $X \in \rho(\Gamma(L))$ as a vector field on the leaf, which is an immersed submanifold. Therefore it makes sense to look at invariance of $\Omega_{L}$ under $X$ :

$$
\begin{aligned}
\mathfrak{L}_{X} \Omega_{L} & \left.=X\rfloor d \Omega_{L}+d(X\rfloor \Omega_{L}\right) \\
& \left.=d(X\rfloor \Omega_{L}\right)=d\left(\left.\omega\right|_{\rho(L)}\right) .
\end{aligned}
$$

The first equality holds because $\Omega_{L}$ is closed, and the second because $\Omega_{L}(X, \cdot)$ $=\left.\omega\right|_{\rho(L)}$ (this is $\omega$ restricted to the leaf). Therefore, $\mathfrak{L}_{X} \Omega_{L}=\left.d \omega\right|_{\rho(L)}$.

Theorem 2.4.2. If $(X, \omega)$ is a section of $L$, then $\mathfrak{L}_{X} \Omega_{L}=\left.d \omega\right|_{\rho(L)}$.

2.5. The bracket on admissible functions. A function $f$ on a Dirac manifold for which $d f \in \rho^{*}(\Gamma(L))$ is called admissible (this is a local condition on $f$ ). If $f$ is admissible then there is a vector field $X_{f}$ such that $\mathbf{e}_{f}=\left(X_{f}, d f\right)$ is a section of $L$. Then if we have two admissible functions $f$ and $g$, we may define their brackets as

$$
\{f, g\}=X_{f} \cdot g \text {. }
$$

Since $\{f, g\}=\Omega_{L}\left(X_{f}, X_{g}\right)$ is antisymmetric, $\{f, g\}$ depends only on $g$ and not on $X_{g}$.

Proposition 2.5.1. The admissible functions form a Poisson algebra.

Proposition 2.5.2. The bracket on admissible functions satisfies the Leibniz identity.

Proof. If $\left(X_{f}, d f\right)$ and $\left(X_{g}, d g\right)$ are sections of $L$, then

$$
g\left(X_{f}, d f\right)+f\left(X_{g}, d g\right)=\left(g X_{f}+f X_{g}, g d f+f d g\right)
$$


is also a section of $L$. Therefore $\left(X_{f g}, d(f g)\right)$ is a section of $L$, where $X_{f g}=g X_{f}+f X_{g}$, which shows that $f g$ is admissible whenever $f$ and $g$ are admissible.

Now we may compute $\{f g, h\}+X_{f g} \cdot h=g X_{f} \cdot h+f X_{g} \cdot h=g\{f, h\}+$ $f\{g, h\}$.

Proposition 2.5.3. If $L$ is an integrable Dirac structure, then the bracket on admissible functions satisfies the Jacobi identity.

Proof. $\mathbf{T}_{L}\left(\mathbf{e}_{f} \otimes \mathbf{e}_{g} \otimes \mathbf{e}_{h}\right)=\left\langle\left[\mathbf{e}_{f}, \mathbf{e}_{g}\right], \mathbf{e}_{h}\right\rangle_{+}$and $\left[\mathbf{e}_{f}, \mathbf{e}_{g}\right]=\left(\left[X_{f}, X_{g}\right], d\{f, g\}\right)$, as is readily verified. Thus we have

$$
\begin{aligned}
\mathbf{T}_{L}\left(\mathbf{e}_{f} \otimes \mathbf{e}_{g} \otimes \mathbf{e}_{h}\right) & =\left\langle\left[\mathbf{e}_{f}, \mathbf{e}_{g}\right], \mathbf{e}_{h}\right\rangle_{+} \\
& =\left\langle\left(\left[X_{f}, X_{g}\right], d\{f, g\}\right),\left(X_{h}, d h\right)\right\rangle_{+} \\
& =\left[X_{f}, X_{g}\right] \cdot h+X_{h} \cdot\{f, g\} \\
& =X_{f} \cdot\{g, h\}-X_{g} \cdot\{f, h\}+\{h,\{f, g\}\} \\
& =\{f,\{g, h\}\}+\{g,\{h, f\}\}+\{h,\{\{f, g\}\} .
\end{aligned}
$$

In the course of this section we have shown

Corollary 2.5.4. If $f$ and $g$ are admissible functions, then so are $f g$ and $\{f, g\}$.

Thus we may prove Proposition 2.5.1.

Proof of 2.5.1. The set of admissible functions is closed under bracket, multiplication, and addition.

2.6. Distributions and leaves. Consider the characteristic distribution of an integrable Dirac structure $L$. As in the linear case, we have

$$
\operatorname{ker} \Omega_{L}=L \cap T P \text {. }
$$

This is the kernel of the smooth bundle map

$$
\rho^{*}(L): L \rightarrow T^{*} P \text {. }
$$

Theorem 2.6.1. If $L \cap T P$ is a bundle, then it is involutive, i.e., it satisfies the Frobenius integrability condition.

To prove this, we use a well-known fact:

Lemma 2.6.2. Let $\alpha$ be a closed 2-form. If the characteristic distribution Char $\alpha$ of $\alpha$ is a subbundle of $T P$, then it is involutive.

Proof. See Abraham and Marsden [1978, p. 298].

Proof of Theorem 2.6.1. We may use the fact that along each leaf we have

$$
\operatorname{ker} \Omega_{L}=L \cap T P .
$$

By virtue of the integrability of $L$ we know that $\Omega_{L}$ is a closed 2-form on each leaf, and therefore by Lemma 2.6.2 $L \cap T P$ is integrable leaf by leaf.

Recall that a Frobenius-integrable subbundle of $T P$ generates a regular foliation. 
Corollary 2.6.3. Suppose that $L \cap T P$ is a subbundle. It is integrable by Theorem 2.6 .1 ; denote its foliation by $\Phi$. Then $P / \Phi$ inherits a Poisson structure from $L$.

Corollary 2.6.4 (well-known). Let $\Omega$ be a closed 2-form on $P$ such that Char $\Omega$ is a bundle; denote its foliation by $\Phi$. Then $P / \Phi$ inherits a symplectic structure. Proof of Corollary 2.6.3. Functions on the manifold $P / \Phi$ may be thought of as functions on $P$ which are constant on $\Phi$, i.e., all $f \in C^{\infty}(P)$ such that $d f(T \Phi)=0$. However these are precisely the admissible functions on $P$.

This shows that functions on the manifold $P / \Phi$ have a bracket which, by integrability of $L$, satisfies the Leibniz and Jacobi identities. This is the induced Poisson structure on $P / \Phi$.

Proof of Corollary 2.6.4. This is the 2-form case of Corollary 2.6.3.

2.7. Hamiltonian systems and equations of motion. A Hamiltonian system is usually defined as a manifold equipped with a bracket on some algebra of functions, together with a choice of function, called the Hamiltonian function. The bracket allows the Hamiltonian function to generate a vector field, called the Hamiltonian vector field. If this vector field is solved for, we say that we have found the equations of motion.

Example 2.7.1. Let $P$ be a Poisson manifold, with bundle map $B: T^{*} P \rightarrow T P$. Then the equations of motion in a local system of coordinates $x^{i}$ are

$$
\dot{x}^{i}=B^{i j} \frac{\partial H}{\partial x^{j}}
$$

and in general we may write the equations of motion as $\dot{x}=X_{H}(x)$, where

$$
X_{H}=B(d H) \text {. }
$$

We will solve for the equations of motion generated by an admissible function of a general integrable Dirac structure. Recall that if we choose local coordinates $x^{i}$ on a neighborhood in $U \subset P$, then a choice of a local basis of sections for $L$ gives us two maps:

$$
\text { a: }\left.L\right|_{U} \rightarrow T P,
$$$$
\text { b: }\left.L\right|_{U} \rightarrow T^{*} P \text {. }
$$

(These maps are just $\rho_{L}$ and $\rho_{L}^{*}$.)

Now let us suppose, as we did in the linear case, that we have an identification $T P \approx T^{*} P$ via a metric; the linear case implies that the sum and difference, $\mathbf{a}+\mathbf{b}$ and $\mathbf{a}-\mathbf{b}$, are invertible at each point. The assumption that $H$ is an admissible function on $P$ implies that there is an $n$-tuple of functions, $\gamma^{i}$, such that

$$
\left(X_{H}\right)^{k} \frac{\partial}{\partial x^{k}}=\gamma^{i} a_{i}^{j} \frac{\partial}{\partial x^{j}}, \quad \frac{\partial H}{\partial x^{k}} d x^{k}=\gamma^{i} b_{i j} d x^{j} .
$$

We write symbolically $X_{H}=\gamma \cdot \mathbf{a}$ and $d H=\gamma \cdot \mathbf{b}$. 
Then we have $X_{H}+d H=\gamma \cdot(\mathbf{a}+\mathbf{b})$ and so $\left(X_{H}+d H\right)(\mathbf{a}+\mathbf{b})^{-1}=\gamma$. But by definition we have $X_{H}=\gamma \cdot \mathbf{a}$ and so $X_{H}=\left(X_{H}+d H\right)(\mathbf{a}+\mathbf{b})^{-1} \mathbf{a}$. Let $\mathbf{C}=(\mathbf{a}+\mathbf{b})^{-1} \mathbf{a} ;$ then this gives us

$$
X_{H}(\mathbf{I}-\mathbf{C})=d H \cdot \mathbf{C} .
$$

But clearly $\mathbf{I}-\mathbf{C}=(\mathbf{a}+\mathbf{b})^{-1} \mathbf{b}$, and so we have

$$
X_{H} \cdot(\mathbf{a}+\mathbf{b})^{-1} \mathbf{b}=d H(\mathbf{a}+\mathbf{b})^{-1} \mathbf{a} .
$$

If $L$ is the graph of a Poisson structure, then $\mathbf{b}$ is invertible and so we may assume that $b$ is the identity. In this case (2.7.6) reads

$$
\begin{gathered}
X_{H} \cdot(\mathbf{a}+\mathbf{I})^{-1} \mathbf{I}=d H(\mathbf{a}+\mathbf{I})^{-1} \mathbf{a}, \\
X_{H}=d H(\mathbf{a}+\mathbf{I})^{-1} \mathbf{a}(\mathbf{a}+\mathbf{I}),
\end{gathered}
$$

and finally

$$
X_{H}=d H \cdot \mathbf{a} .
$$

Equation (2.7.8) is exactly the system of equations given in (2.7.2). In fact, abandoning the shorthand, we have

$$
\dot{x}^{j} \frac{\partial}{\partial x^{j}}=\frac{\partial H}{\partial x^{i}} \mathbf{a}^{i j} \frac{\partial}{\partial x^{j}},
$$

which is exactly (2.7.1), where the matrix a has taken the place of the bundle map $B$, and the first index has been raised using the suppressed isomorphism between tangent and cotangent bundles.

In general, the equations of motion have the form of (2.7.6):

$$
B_{i j} \dot{x}^{j}=A_{i}^{j} \frac{\partial H}{\partial x^{j}} .
$$

If we adapt the system of coordinates to the kernels of the maps $a$ and $\mathbf{b}$, we get the following general system of equations:

(2.7.11a) (1) $\alpha_{i j} \dot{x}^{j}=0 \quad$ (equations of constraint; constants of the motion),

$$
\text { (2) } \beta_{i}^{j} \frac{\partial H}{\partial x^{j}}=0 \quad \text { (condition on admissibility of } H \text {; }
$$

gauge equations),

$$
\text { (3) } \dot{x}^{j}=\nu^{i j} \frac{\partial H}{\partial x} \quad \text { (equations of motion; dynamics) }
$$

These are the general equations of constrained dynamics.

\section{Constrained Dirac structures}

3.1. Dirac reduction on manifolds. We will now apply the process outlined in $\S 1.4$ to almost Dirac structures: maximal isotropic subbundles of $T P \oplus T^{*} P$ 
under the pairing $\langle,\rangle_{+}$. Let $Q$ be a smooth submanifold of $P$. Then we may define

$$
L_{Q}=\frac{L \cap\left(T Q \oplus T^{*} P\right)}{L \cap T Q^{\circ}}
$$

At each point of $Q$ this is a Lagrangian subspace of $T Q \oplus T^{*} Q$. If $L_{Q}$ happens to be a smooth subbundle of $T Q \oplus T^{*} Q$ then we have an almost Dirac structure on $Q$. Notice that $L \cap\left(\{0\} \oplus T Q^{\circ}\right)$ may be considered as a subset of $L$ or of $T^{*} P$; from now on we will write $L \cap T Q^{\circ}$ in place of $L \cap\left(\{0\} \oplus T Q^{\circ}\right)$.

Now $L \cap\left(T Q \oplus T^{*} P\right)$ is a subbundle if and only if it is of constant dimension, and since the quotient has constant dimension this happens if and only if $L \cap$ $T Q^{\circ}$ has constant dimension. Finally, $L_{Q}$ is a smooth bundle if both the numerator and denominator are bundles. Thus we have

Theorem 3.1.1. The following are equivalent:

(1) $L \cap\left(T Q \oplus T^{*} P\right)$ has constant dimension.

(2) $L \cap T Q^{\circ}$ has constant dimension.

Furthermore, if either of the above hold, then $L_{Q}$ is an almost Dirac structure on $Q$.

Definition 3.1.2. If the conditions of Theorem 3.1 .1 hold, then we call $Q$ a clean submanifold of $P$ (relative to $L$ ). Thus if $Q$ is a clean submanifold, $L \cap\left(T Q \oplus T^{*} P\right)$ and $L \cap T Q^{\circ}$ are subbundles of $L$.

If $Q$ is a clean submanifold of $P$, then we may realize sections of $L_{Q}$ as sections of the bundle $L \cap\left(T Q \oplus T^{*} P\right)$ modulo sections of $L \cap T Q^{\circ}$. This has the happy consequence that the integrability tensors of $L$ and $L_{Q}$ are intertwined.

Proposition 3.1.3. Let $i: L \cap\left(T Q \oplus T^{*} P\right) \rightarrow L$ be the inclusion map, and let $\pi_{Q}: L \cap\left(T Q \oplus T^{*} P\right) \rightarrow L_{Q}$ be the bundle map whose kernel is $L \cap T Q^{\circ}$. Then we have

$$
\pi_{Q}^{*} \mathbf{T}_{L_{Q}}=i^{*} \mathbf{T}_{L}
$$

Corollary 3.1.4. Let $Q$ be clean. If $L$ in integrable, then $L_{Q}$ is integrable.

Proof of Proposition 3.1.3. According to the remark preceding the proposition, if we are given a section $(X, \omega)$ of $L_{Q}$ so that $X \in \Gamma(T Q)$ and $\omega \in \Gamma\left(T^{*} Q\right)$, then we may find a section $(X, \tilde{\omega})$ of $L$ such that $\left.\tilde{\omega}\right|_{T Q}=\omega$. Now suppose that we have three sections of $L_{Q}$, say $(X, \tilde{\omega}),(Y, \tilde{\mu})$, and $(Z, \tilde{\nu})$; then the 
integrability 3-tensor on $Q$ evaluated on these sections equals

$$
\begin{aligned}
\mathbf{T}_{L_{Q}}((X, \omega) \oplus(Y, \mu) \otimes(Z, \nu)) & \\
=-\frac{1}{2}(\omega \cdot[Y, Z]+\mu \cdot[Z, X]+ & \nu \cdot[X, Y]+X \cdot \mu(Z) \\
& +Y \cdot \nu(X)+Z \cdot \omega(Y)) \\
=-\frac{1}{2}(\tilde{\omega} \cdot[Y, Z]+\tilde{\mu} \cdot[Z, X]+\tilde{\nu} \cdot[X, Y]+X \cdot \tilde{\mu}(Z) & +Y \cdot \tilde{\nu}(X)+Z \cdot \tilde{\omega}(Y)) \\
= & \mathbf{T}_{L}((X, \tilde{\omega}) \otimes(Y, \tilde{\mu}) \otimes(Z, \tilde{\nu})) .
\end{aligned}
$$

This last expression is exactly $i^{*} \mathbf{T}_{L}((X, \tilde{\omega}) \otimes(Y, \tilde{\mu}) \otimes(Z, \tilde{\nu}))$, whereas the first expression is $\pi_{Q}^{*} \mathbf{T}_{L_{Q}}((X, \tilde{\omega}) \otimes(Y, \tilde{\mu}) \otimes(Z, \tilde{\nu}))$. This establishes (3.1.2).

Proof of Corollary 3.1.4. Use the fact that $\pi_{Q}$ is a surjection.

Proposition 3.1.5. If $Q \subset P$, then we have

$$
L_{Q} \cap T Q \approx L \cap T Q \oplus T Q^{\circ} / L \cap T Q^{\circ} .
$$

Proof. We compute

$$
\begin{aligned}
L_{Q} \cap T Q & \approx\left\{(X, 0) \in L_{Q} \mid X \in T Q\right\} \\
& \approx\left\{(X, \omega) \in L \mid X \in T Q \text { and }\left.\omega\right|_{T Q}=0\right\} / L \cap T Q^{\circ} \\
& \approx\left\{(X, \omega) \in L \mid X \in T Q \text { and } \omega \in T Q^{\circ}\right\} / L \cap T Q^{\circ} \\
& \approx L \cap\left(T Q \oplus T Q^{\circ}\right) / L \cap T Q^{\circ}
\end{aligned}
$$

at each point. This establishes (3.1.3).

We have seen conditions under which a submanifold $Q$ inherits a Dirac structure. The leaves of $Q$ are the intersections of the leaves of $P$ with $Q$, and the 2-forms on the leaves are the restrictions to $Q$ of the 2-forms on the leaves of $P$. However the formula above shows that the characteristic distribution of the induced structure is not so obvious; this is because it depends on how $Q$ intersects the leaves of $P$ and the characteristic distribution of $P$.

3.2. Reduction in the Poisson case. Suppose $P$ is a Poisson manifold with structure determined by the skew bundle map $B: T^{*} P \rightarrow T P$. In this case, the conditions of Theorem 3.1.1 work as follows:

$$
\begin{aligned}
L \cap T Q^{\circ} & \approx\left\{(0, \omega) \in L \mid \omega \in T Q^{\circ}\right\} \\
& \approx\left\{\omega \in T Q^{\circ} \mid \omega \in \operatorname{ker} B\right\} \approx T Q^{\circ} \cap \operatorname{ker} B .
\end{aligned}
$$

Now $L \cap T Q^{\circ}$ is a subbundle of $T^{*} P$ if and only if its orthogonal complement is a subbundle of $T P$, i.e.,

$$
\left(T Q^{\circ} \cap \operatorname{ker} B\right)^{\circ}=T Q+(\operatorname{ker} B)^{\circ}=T Q+\operatorname{Im} B
$$

is a subbundle of $T P$; thus $Q$ is clean if $T Q+\operatorname{Im} B$ is a bundle. 
Our second condition is that $L \cap\left(T Q \oplus T^{*} P\right)$ has constant dimension. In the Poisson case this is

$$
L \cap\left(T Q \oplus T^{*} P\right) \approx\{(B(\omega), \omega) \in L \mid B(\omega) \in T Q\} \approx \operatorname{Im} B \cap T Q .
$$

Once again looking at orthogonal complements, we get

$$
(\operatorname{Im} B \cap T Q)^{\circ} \approx T Q^{\circ}+(\operatorname{Im} B)^{\circ} \approx T Q^{\circ}+\operatorname{ker} B,
$$

so this condition may be read as saying that there are locally a constant number of independent Casimir constraints (functions which are Casimir and constant on $Q$ ). Now,

$$
L_{Q} \cap T Q \approx L \cap\left(T Q \oplus T Q^{\circ}\right) \approx T Q \cap B\left(T Q^{\circ}\right),
$$

which, as is pointed out in Weinstein [1983], is the kernel of the restricted 2 -form. Notice that in general this does not have to be a bundle.

As in Weinstein [1983] we will state sufficient conditions for a submanifold $Q$ of a Poisson manifold $P$ to inherit a Poisson structure:

Theorem 3.2.1. Suppose the following conditions hold:

(a) $\operatorname{ker} B \cap T Q^{\circ}$ is a bundle.

(b) $T Q \cap B\left(T Q^{\circ}\right)=\{0\}$.

Then $L_{Q}$ defines a Poisson structure on $Q$.

The similar condition in Weinstein [1983] is that $\operatorname{ker} B \cap T Q^{\circ}=\{0\}$.

If $P$ is a Poisson manifold, submanifolds $Q$ which are transverse to any leaf of $P$ satisfy these conditions and inherit a Poisson structure. This is because $T Q+\operatorname{Im} B=T P$ is a bundle and therefore satisfies condition (1) of Theorem 3.1.1. Suppose now that we have a submanifold $Q$ of a Dirac manifold $P$, and that $T_{x} Q \oplus \rho(L)=T_{x} P$ holds at $x$; then $T Q+\rho(L)=T P$ locally for the same reasons as in the Poisson case. This implies that

$$
\begin{aligned}
\{0\} & =T P^{\circ}=(T Q+\rho(L))^{\circ}=T Q^{\circ} \cap \rho(L)^{\circ} \\
& =T Q^{\circ} \cap\left(L \cap T^{*} P\right)=L \cap T Q^{\circ} .
\end{aligned}
$$

Therefore by Definition 3.1.2 submanifolds transverse to leaves are clean: they inherit natural Dirac structures. However, whether or not different transverse manifolds are locally isomorphic as they are in the Poisson case remains an open question.

3.3. Momentum level sets as Dirac manifolds. Let $L \subset T P \oplus T^{*} P$ be the graph of a Poisson bundle map $B: T^{*} P \rightarrow T P$, and suppose that a Lie group $G$ acts on $P$ by Poisson automorphisms so that $\mathfrak{g}$ generates locally Hamiltonian vector fields. Finally assume that we have an equivariant momentum map $J: P \rightarrow \mathfrak{g}^{*}$ and let $Q=J^{-1}(\mu)$; then $T_{x} Q=\operatorname{ker} T_{x} J$ and $\left(T_{x} Q\right)^{\circ}=\left(\operatorname{ker} T_{x} J\right)^{\circ}=$ $\operatorname{Im}\left(T_{x} J\right)^{*}$. The map $\left(T_{x} J\right)^{*}$ satisfies the property $B_{x}\left(T_{x} J\right)^{*} \cdot \xi=\xi_{P}(x)$. This is interpreted as follows: since the vector fields generated by $\mathfrak{g}$ are locally Hamiltonian they have Hamiltonian functions; thus we get a map $\xi \rightarrow H_{\xi}$ which 
satisfies $\left(T_{x} J\right)^{*} \cdot \xi=d H_{\xi}(x)$. Let us define

$$
S_{x}=\left\{\xi \in \mathfrak{g} \mid d H_{\xi}(x)=0\right\} .
$$

Finally, recall that $T_{x} J \cdot \xi_{P}(x)=\operatorname{ad}_{\xi}^{*} \mu$, so that $\xi_{P}(x)$ is tangent to $Q$ implies that $\xi \in \mathfrak{g}_{\mu}$.

Now let us examine the condition that $L \cap T Q^{\circ}$ be a bundle, i.e., that the dimension of $L \cap T_{x} Q^{\circ}$ be locally constant. We have

$$
\begin{aligned}
L \cap T Q^{\circ} & =\left\{\left(0,\left(T_{x} J\right)^{*} \cdot \xi\right) \in L \mid \xi \in \mathfrak{g}\right\} \\
& =\left\{\left(0, d H_{\xi}\right) \in L \mid \xi \in \mathfrak{g}\right\} \approx\left\{\xi \mid \xi_{P}(x)=0\right\} / S_{x}=\mathfrak{g}_{x} / S_{x} .
\end{aligned}
$$

So $L \cap T_{x} Q^{\circ} \approx \mathfrak{g}_{x} / S_{x}$. This is locally constant if and only if $x$ is on an orbit of principal type. Therefore $Q=J^{-1}(\mu)$ is a clean submanifold in a neighborhood of $x \in J^{-1}(\mu)$ on a principal orbit, and $L_{J^{-1}(\mu)}$ becomes a smooth Dirac structure on $J^{-1}(\mu)$. The characteristic distribution of this structure is given locally by

$$
L_{Q} \cap T_{x} Q \approx L \cap\left(T_{x} Q \oplus T_{x} Q^{\circ}\right) / L \cap T_{x} Q^{\circ}
$$

and we have

$$
\begin{aligned}
& L \cap\left(T_{x} Q \oplus T_{x} Q^{\circ}\right)=L \cap\left(T_{x} Q \oplus \operatorname{Im}\left(T_{x} J\right)^{*}\right) \\
& \quad \approx\left\{(X, \omega) \in L \mid X=B(\omega) \in T_{x} Q, \omega=d H_{\xi}(x) \text { for some } \xi \in \mathfrak{g}\right\} \\
& \quad \approx\left\{\xi_{P^{(x)}} \mid \xi \in \mathfrak{g}_{\mu}\right\} / S_{x},
\end{aligned}
$$

so $L_{Q} \cap T_{x} Q \approx \mathfrak{g}_{\mu} / \mathfrak{g}_{x}$, which shows that $L_{Q} \cap T Q$ is a subbundle of $T Q$ whose integral manifolds are $G_{\mu}$ orbits. The action of $G_{\mu}$ on $J^{-1}(\mu)$ clearly preserves $L_{J^{-1}(\mu)}$, so there is a Dirac structure induced on $J^{-1}(\mu) / G_{\mu}$ whose characteristic distribution is zero. This is the reduced Poisson structure.

\section{EXAMPLES}

4.1. Regular points and local structure. Recall the characteristic equations of a Dirac structure:

$$
\begin{aligned}
& \rho(L)^{\circ}=L \cap T^{*} P, \\
& \rho^{*}(L)=(L \cap T P)^{\circ} .
\end{aligned}
$$

It follows from these equations that $\rho(L)$ has maximal dimension exactly when $L \cap T^{*} P$ has minimal dimension, and that $\rho^{*}(L)$ has maximal dimension exactly when $L \cap T P$ has minimal dimension.

Now since $\rho$ is a smooth bundle map there is an open dense set on which $\rho(L)$ has maximal dimension; observe that $\rho(L)$ is a bundle over this set, and thus $L \cap T^{*} P$ is also a bundle over this set. In the same way, we find another open dense set on which $\rho^{*}(L)$ and $L \cap T P$ are both bundles. 
Definition 4.1.1. The open dense set on which the characteristic equations of a Dirac structure are bundle equations is called the set of regular points of the Dirac structure. Thus a point is regular if there is a neighborhood of the point over which the quantities $\rho(L), L \cap T P, \rho^{*}(L)$, and $L \cap T^{*} P$ are all bundles.

Recall that the foliation generated by $L \cap T P$ is denoted by $\Phi$, and that the (local) manifold $P / \Phi$ has a Poisson structure. We will now see that any manifold strictly transverse to $L \cap T P$ has a Poisson structure. For information on transverse Poisson structures, see Weinstein [1983], Oh [1986], and Montgomery [1985].

Let $Y$ be a submanifold of $P$ transverse to $L \cap T P$, i.e.,

$$
\begin{aligned}
& T Y \oplus(L \cap T P)=T P, \\
& T Y \cap(L \cap T P)=0 .
\end{aligned}
$$

Forming annihilators of these quantities establishes the additional formulas:

$$
\begin{aligned}
& T Y^{\circ}+(L \cap T P)^{\circ}=T P, \\
& T Y^{\circ} \cap(L \cap T P)^{\circ}=0 .
\end{aligned}
$$

Using the fact that $(L \cap T P)^{\circ}=\rho^{*}(L),(4.1 .3 \mathrm{a}, \mathrm{b})$ may be written as

$$
T Y^{\circ} \oplus \rho^{*}(L)=T^{*} P .
$$

Since $L \cap T^{*} P \subset \rho^{*}(L),(4.1 .4)$ implies that

$$
L \cap T Y^{\circ}=0 .
$$

Therefore $Y$ is a clean submanifold of $P$ (relative to $L$ ). In fact the integrable Dirac structure $L_{Y}$ is given by

$$
L_{Y} \approx L \cap\left(\left.T Y \oplus T^{*} P\right|_{T Y}\right) .
$$

We will now determine the characteristic distribution of $L_{Y}$. Since $L \cap T Y^{\circ}=0$ we have

$$
\begin{aligned}
L_{Y} \cap T Y & \approx L \cap\left(T Y \oplus T Y^{\circ}\right) \\
& \approx\left\{(X, \omega) \in L \mid X \in T Y \text { and } \omega \in T Y^{\circ}\right\} \\
& \approx\{(X, 0) \in L \mid X \in T Y\} \quad\left(\text { since } T Y^{\circ} \cap \rho^{*}(L)=0\right) \\
& \approx 0 \quad(\text { since } T Y \cap(L \cap T P)=0) .
\end{aligned}
$$

Therefore $L_{Y}$ is actually a Poisson structure on $Y$.

By Theorem 2.6.1, $L \cap T P$ is an integrable bundle in a neighborhood of a regular point and therefore we may find coordinates $(x, y)$ such that

$$
\left(\frac{\partial}{\partial x^{1}}, 0\right), \ldots,\left(\frac{\partial}{\partial x^{r}}, 0\right)
$$

are a basis of sections for $L \cap T P$. If we consider the functions $x^{i}$ as constraints, then the discussion above shows that the manifolds given by level sets of the $x^{i}$ 's are all Poisson manifolds with Poisson structure given by (4.1.6). 
In fact, since the coordinate functions $y^{j}$ are all admissible functions, we may write a local basis of sections for $L$ in these coordinates

$$
\left(\partial / \partial x^{i}, 0\right), \ldots,\left(\xi_{y^{j}}, d y^{j}\right) .
$$

Thus the bundle $L \cap\left(T y \oplus T^{*} P\right)$ has local sections given by

$$
\left(\xi_{y^{j}}, d y^{j}\right) \text {, }
$$

along each slice $Y=\{x=$ constant $\}$. Hence the structure $L_{Y}$ is Poisson and is given as follows: chose two functions on $Y=\{x=$ constant $\}$, extend them to admissible functions on $P$, and compute the bracket on admissible functions, i.e., we identify each slice $Y=\{x=$ constant $\}$ with the Poisson manifold $P / \Phi$. Now fix an admissible function $q$. Since the manifold $P / \Phi$ is Poisson, there is an admissible function $p$ such that the bracket on admissible functions is

$$
\{q, p\}=1 .
$$

Thus we may perform the Darboux algorithm on the algebra of admissible functions. Therefore we may find coordinates $(x, q, p, c)$ such that $(q, p, c)$ are Darboux coordinates on the slice $Y=\{x=$ constant $\}$. Thus we have shown:

Proposition 4.1.2. In a neighborhood of a regular point on an integrable Dirac manifold we may find coordinates $(x, q, p, c)$ such that a local basis of sections for the Dirac structure is given by

$$
\left(\frac{\partial}{\partial x^{i}}, 0\right), \ldots,\left(\frac{\partial}{\partial p_{r}}, d q^{r}\right), \ldots,\left(-\frac{\partial}{\partial q^{s}}, d p_{s}\right), \ldots,\left(0, d c^{k}\right) .
$$

The $x$ 's are called characteristic coordinates, the $q$ 's and $p$ 's are called canonical coordinates, and the $c$ 's are called Casimirs.

Definition 4.1.3. We shall call such coordinates Darboux coordinates.

Lemma 4.1.4. In Darboux coordinates the restriction of $\langle,\rangle_{-}$to $L$ is given by

$$
\begin{gathered}
\left(\begin{array}{cccc}
0 & 0 & 0 & 0 \\
0 & 0 & -\mathbf{I} & 0 \\
0 & \mathbf{I} & 0 & 0 \\
0 & 0 & 0 & 0
\end{array}\right) . \\
x \text { 's } q \text { 's } p \text { 's } c \text { c's }
\end{gathered}
$$

4.2. Jacobi structures at regular points. An integrable Jacobi structure on a manifold is a pair $(\Lambda, E)$, where $\Lambda$ is a bivector field and $E$ is a vector field satisfying

$$
\begin{aligned}
& {[\Lambda, \Lambda]=2 E \wedge \Lambda,} \\
& {[E, \Lambda]=0 .}
\end{aligned}
$$


Given a function $u$ on $P$ we define

$$
X_{u}=\Lambda\lfloor d u+u E
$$

$X_{u}$ is called the Hamiltonian vector field generated by the Jacobi structure. For a discussion of Jacobi structures see Lichnerowicz [1977].

We will show that in a neighborhood of a point where $E \neq 0$, a Jacobi structure is actually an integrable Dirac structure with characteristic distribution $L \cap T P=\operatorname{span}(E)$. This condition determines the set of admissible functions, namely all $u \in C^{\infty}(P)$ such that $d u \in E^{\circ}$. In addition we will have the distribution $\rho(L)=\Lambda\left(E^{\circ}\right) \oplus \operatorname{span}(E)$.

Let $u^{1}, \ldots, u^{n-1}$ be independent admissible functions. Then we may write a local basis of sections for an almost Dirac structure $L$ :

$$
\left(\Lambda\left\lfloor d u^{1}, d u^{1}\right), \ldots,\left(\Lambda\left\lfloor d u^{n-1}, d u^{n-1}\right), \ldots,(E, 0) .\right.\right.
$$

By adding multiples of the section $(E, 0)$ we get another local basis for $L$ :

$$
\left(X_{u^{1}}, d u^{1}\right), \ldots,\left(X_{u^{n-1}}, d u^{n-1}\right), \ldots,(E, 0) .
$$

Therefore the Hamiltonian vector fields generated by admissible functions are the Hamiltonian vector fields generated by the Jacobi structure. Thus the bracket on admissible functions is given by

$$
\{u, v\}=X_{u} \cdot v=\Lambda\lfloor d u \wedge d v+u E \cdot v=\Lambda\lfloor d u \wedge d v .
$$

We now compute the integrability tensor $T_{L}$ of the almost Dirac structure defined by the local sections given by (4.2.3) and (4.2.4). Let $f, g$, and $h$ be admissible functions and let $\mathbf{e}_{f}=\left(X_{f}, d f\right)$ denote their admissible sections:

$$
\begin{aligned}
\mathbf{T}_{L}\left(\mathbf{e}_{f} \otimes \mathbf{e}_{g} \otimes \mathbf{e}_{h}\right) & =\left\langle\left[\mathbf{e}_{f}, \mathbf{e}_{g}\right], \mathbf{e}_{h}\right\rangle_{+} \\
& =\{f,\{g, h\}\}+\{h,\{f, g\}\}+\{g,\{h, f\}\} \\
& =\left\langle\frac{1}{2}[\Lambda, \Lambda] \mid d f \wedge d g \wedge d h\right\rangle \\
& =\langle E \wedge \Lambda \mid d f \wedge d g \wedge d h\rangle=0
\end{aligned}
$$

since all the functions annihilate $E$.

In addition we have

$$
\begin{aligned}
\mathbf{T}_{L}\left(\mathbf{e}_{f} \otimes \mathbf{e}_{g} \otimes(E, 0)\right) & =\left\langle\left[\mathbf{e}_{f}, \mathbf{e}_{g}\right],(E, 0)\right\rangle_{+} \\
& =\left\langle\left(\left[X_{f}, X_{g}\right],\{d f, d g\}\right),(E, 0)\right\rangle_{+} \\
& =E \cdot\{f, g\}=E \cdot(\Lambda\lfloor d f \wedge d g) \\
& =[E, \Lambda]\lfloor d f \wedge d g=0
\end{aligned}
$$

using equation $(4.2 .1 \mathrm{~b})$ and the fact that both functions are admissible. Therefore $L$ is a Dirac structure.

Notice that for (4.2.3) and (4.2.4) to define a maximally isotropic subbundle of $T P \oplus T^{*} P$ we must have $E \neq 0$. It seems unlikely that this example can be extended to a neighborhood where $E=0$, as a result of the condition $L \cap T P=E ; L \cap T P$ is a kernel of a bundle map and thus has minimal rank locally on an open dense set, whereas the distribution defined by $E$ is maximal locally on an open dense set. 


\section{REFERENCES}

R. Abraham and J. Marsden [1978], Foundations of mechanics, 2nd ed., Addison-Wesley, Reading, Mass.

Gloria Alvarez-Sanchez [1986], Geometric methods of classical mechanics applied to control theory, Ph.D. thesis, University of California at Berkeley.

Coste, Dazord and Weinstein [1986], (to appear).

Pierre Dazord [1985], Feuilletages à singularités, Nederl. Akad. Wetensch. Proc. Ser. A 88, 21-39.

P. A. M. Dirac [1964], Lectures in quantum mechanics, Yeshiva University.

M. J. Gotay and J. E. Nester [1979], Ann. Inst. H. Poincaré Anal. Non Linéaire 30, 129.

M. J. Gotay [1983], Coisotropic imbeddings, Dirac brackets and quantization, preprint.

M. J. Gotay, J. E. Nester, and G. Hinds [1978], Presymplectic manifolds and the Dirac theory of constraints, J. Math. Phys. 19, 2388-2399.

V. Guillemin and S. Sternberg [1977], Geometric asymptotics, Math. Surveys, no. 14, Amer. Math. Soc., Providence, R.I.

A. J. Hanson, T. Regge, and C. Teitelboim [1976], Accad. Naz. Lincei Rome 22.

R. Hermann [1970], Lie algebras and quantum mechanics, Benjamin, New York.

André Lichnerowicz [1977], Les variétés de Poisson et leurs algèbres de Lie associées, J. Differential Geometry 12, 253-300.

R. G. Littlejohn [1979], A guiding center Hamiltonian : A new approach, J. Math. Phys. 20, 24452458.

[1981], Hamiltonian formulation of guiding center motion, Phys. Fluids 24, 1730-1749.

K. Mackenzie [1987], Lie groupoids and Lie algebroids in differential geometry, Lecture Notes Series, no. 134, London Math. Soc., Cambridge Univ. Press.

J. Marsden and T. Ratiu [1985], Reduction of Poisson manifolds, Lett. Math. Phys. 11, 161-170.

Jean Martinet [1970], Sur les singularités des formes différentielles, Ann. Inst. Fourier (Grenoble), 20, 95-178.

Richard Montgomery [1985], personal communication.

Yong-Geun Oh [1986], Some remarks on the transverse Poisson structures of coadjoint orbits, Lett. Math. Phys. 12, 87-91.

S. Omohundro [1984], Geometric Hamiltonian structures and perturbation theory, in Local and Global Methods in Nonlinear Dynamics (B. Cawley, ed.), Lecture Notes in Phys., SpringerVerlag, New York.

- [1985], Geometric perturbation theory and plasma physics, Ph.D. thesis, University of California at Berkeley.

Spyros N. Pnevmatikos [1985], Évolution dynamique d'un système mécanique en présence de singularités génériques, Singularities and Dynamical Systems, North-Holland, pp. 209-217.

[1979], Structures hamiltoniennes en présence de contrainte, C. R. Acad. Sci. Paris Sér. I Math. 289, 799-802.

_ [1984], Structures symplectiques singulières génériques, Ann. Inst. Fourier (Grenoble) 34, 201218.

J. Sniatycki [1974], Dirac brackets in geometric dynamics, Ann. Inst. H. Poincaré, Anal. Non Linéaire 20, 365-372.

Héctor Sussman [1973], Orbits of families of vector fields and integrability of distributions, Trans. Amer. Math. Soc. 180, 171-188.

A. M. Vinogradov and I. S. Krasilshchik [1975], Russian Math. Surveys 30, 177-202.

A. Weinstein [1983], The local structure of Poisson manifolds, J. Differential Geometry 18, 523-557.

A. Weinstein and J. Sniatycki [1983], Reduction and quantization for singular momentum mappings, Lett. Math. Phys. 7, 115-161. 